Florida International University FIU Digital Commons

FIU Electronic Theses and Dissertations

University Graduate School

5-19-2014

\title{
Overnight Summer Music Camp and the Impact on its Youth: A Case Study
}

Ari Nemser

Florida International University, anems001@fiu.edu

DOI: $10.25148 /$ etd.FI14071188

Follow this and additional works at: https://digitalcommons.fiu.edu/etd

\section{Recommended Citation}

Nemser, Ari, "Overnight Summer Music Camp and the Impact on its Youth: A Case Study" (2014). FIU Electronic Theses and Dissertations. 1551.

https://digitalcommons.fiu.edu/etd/1551

This work is brought to you for free and open access by the University Graduate School at FIU Digital Commons. It has been accepted for inclusion in FIU Electronic Theses and Dissertations by an authorized administrator of FIU Digital Commons. For more information, please contact dcc@fiu.edu. 


\title{
FLORIDA INTERNATIONAL UNIVERSITY
}

Miami, Florida

OVERNIGHT SUMMER MUSIC CAMP AND THE IMPACT ON ITS YOUTH: A CASE STUDY

\author{
A thesis submitted in partial fulfillment of the \\ requirements for the degree of \\ MASTER OF SCIENCE \\ in \\ MUSIC EDUCATION \\ by
}

Ari Nemser

2014 


\section{To: Dean Brian Schriner}

College of Architecture and the Arts

This thesis, written by Ari Nemser, and entitled Overnight Summer Music Camp and the Impact on its Youth: A Case Study, having been approved in respect to style and intellectual content, is referred to you for judgment.

We have read this thesis and recommend that it be approved.

Cathy Benedict

David Dolata

Patrick Schmidt, Major Professor

Date of Defense: May 19, 2014

The thesis of Ari Nemser is approved.

Dean Brian Schriner

College of Architecture and the Arts

Dean Lakshmi N. Reddi

University Graduate School

Florida International University, 2014 


\section{ACKNOWLEDGMENTS}

I would like to express my deepest gratitude to several people who have had a deep impact on my life as a musician, educator, and individual.

Dr. Patrick Schmidt: Your guidance in my path as a music educator and scholar has truly been incredible. I thank you for the patience you have shown me throughout my graduate career as well as the never ending wealth of constructive feedback in all my academic endeavors. You have helped me find my voice as a writer and critical thinker.

Dr. Cathy Benedict: You have shown me how to open my eyes, engaging critically with the world and realizing potentiality beyond musical engagements. You have helped me discover that successes in life are worth more than just a good job, but worthy of meaningful recognition.

Dr. David Dolata: I am most grateful for your professional support during my timeat FIU. Your guidance has greatly impacted both my writing and my outlook as a music educator.

Dr. Joel Galand: Your support during my time at FIU as you assisted me throughout the process with great care and professionalism is greatly appreciated and remembered. Professor Jim Hacker: My mentor and friend. This journey began over twenty years ago when I heard the sound of the trumpet. You have shown tremendous dedication to my success as a trumpet player, helping me discover what it means to overcome challenging obstacles through perseverance.

Mom and Dad: Your unconditional love and support is an unmeasurable blessing. Your Lion of Judah is now ready to sound the trumpet throughout the land and among the nations. 
JacoboNitsch: Mi compadre. Throughout the journey of graduate school your musical support, moral support, and most of all friendship, has been more meaningful than I can express, and I am grateful to have made such an amazing lifelong friend.

Jamie and Ellen Saltman: Your dedication to sharing the music camp experience is a beautiful thing, impacting so many lives in such meaningful ways. I am grateful and privileged to have been a part of it. 


\section{ABSTRACT OF THE THESIS \\ OVERNIGHT SUMMER MUSIC CAMP AND THE IMPACT ON ITS YOUTH: A \\ CASE STUDY \\ by}

Ari Nemser

Florida International University, 2014

Miami, Florida

\section{Professor Patrick Schmidt, Major Professor}

This qualitative case study explores an overnight music camp, Camp Encore

Coda. The purpose is to gain an understanding of the environment, uncovering the unique ways it impacts the social and musical developments of its participating youth while contrasting it to that of the traditional classroom. Framed by the concept of multidimensional growth, data were collected using surveys, interviews, focus group, and observations. The study uncovered exorbitant levels of social and musical growth among the youth; all directly linked to isolation from technology, communal living, and musical immersion in a community of practice. There lacks a significant amount of music education research attempting to explore and provide an initial evaluation of the learning opportunities unique to overnight music camps. This is particularly significant for music educators who strive to continue the advancement of the field through positive impact on students inside and outside of the school classroom. 


\section{TABLE OF CONTENTS}

CHAPTER

PAGES

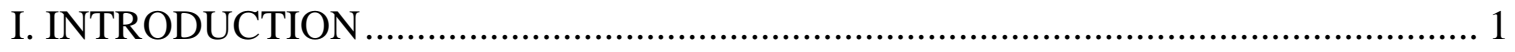

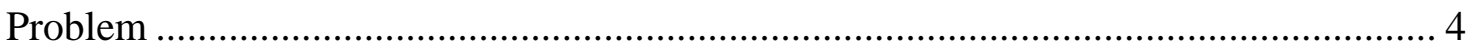

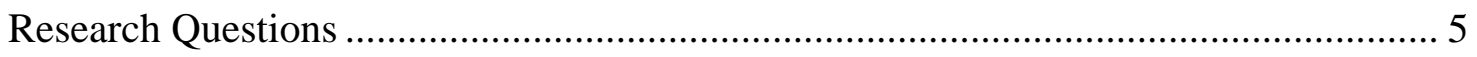

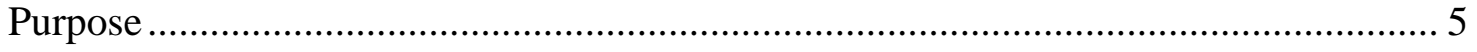

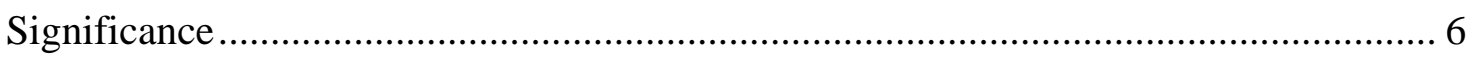

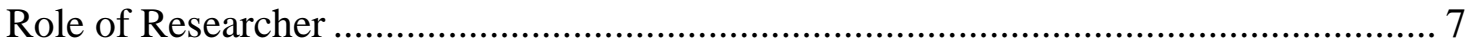

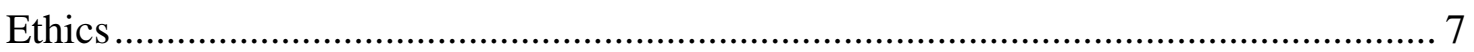

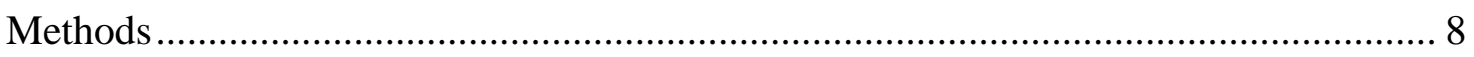

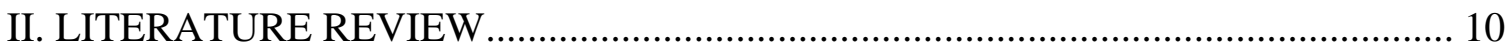

The Camp Environment .................................................................................... 10

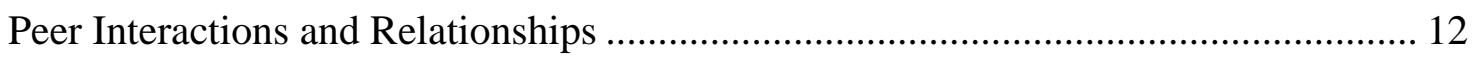

Traditional Classroom .................................................................................. 14

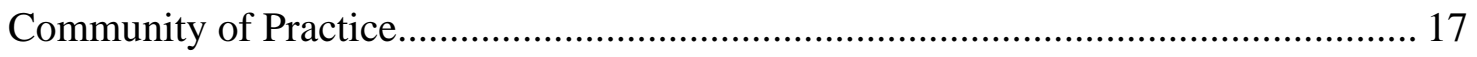

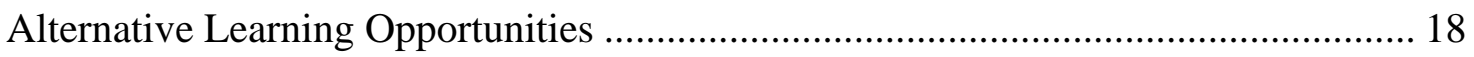

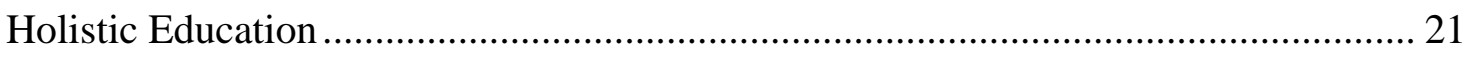

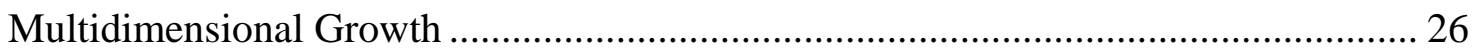

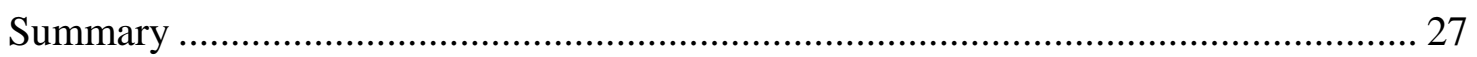

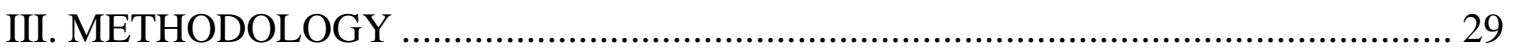

Rationale for Qualitative Design....................................................................... 29

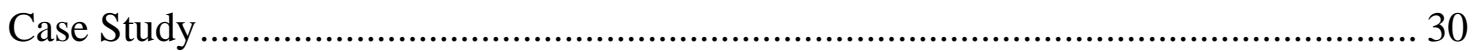

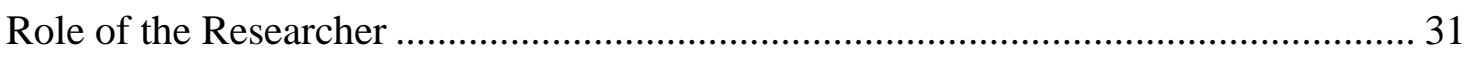

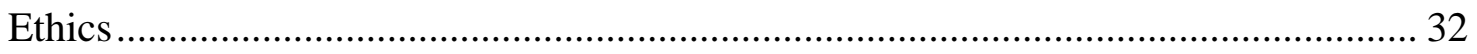

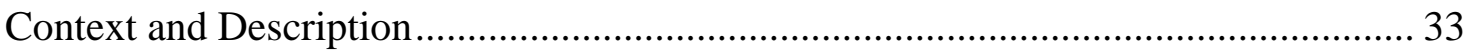

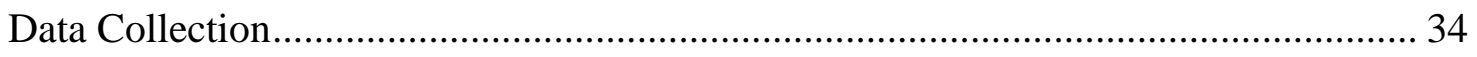

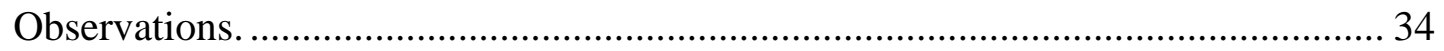

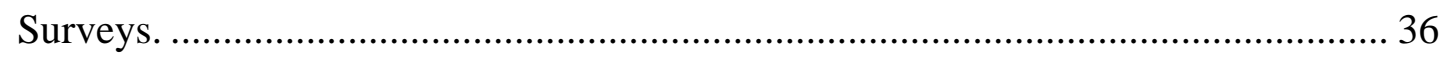

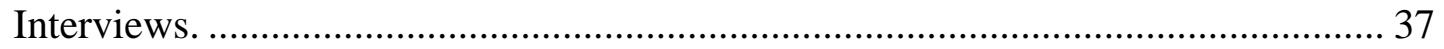

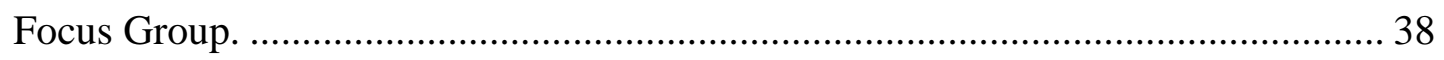

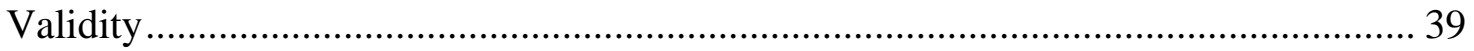

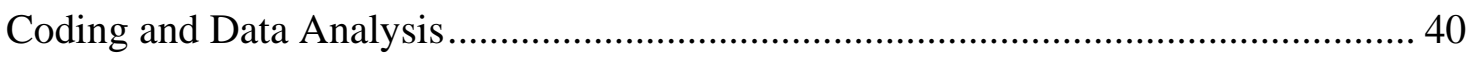

Limitations and De-limitations................................................................................... 41

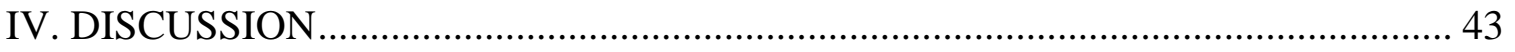

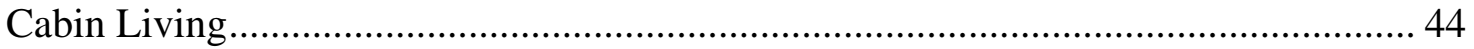

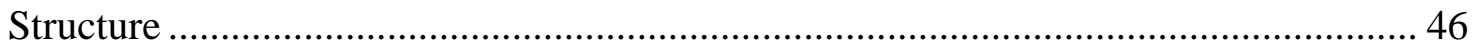




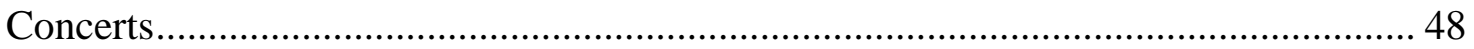

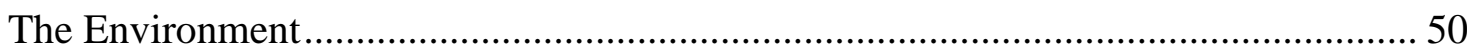

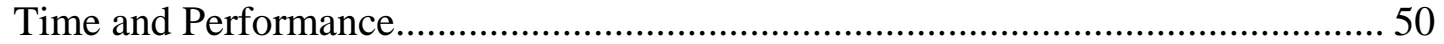

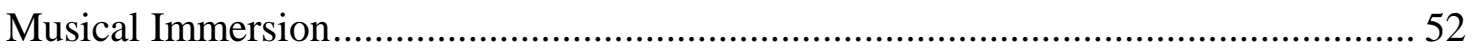

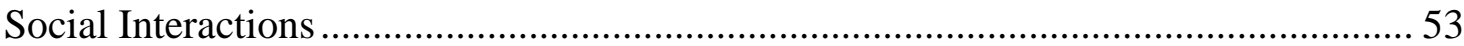

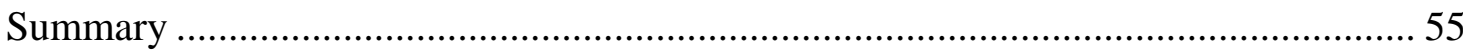

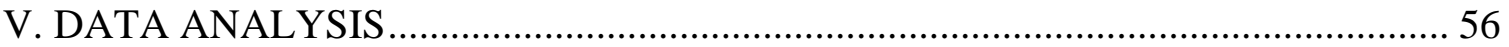

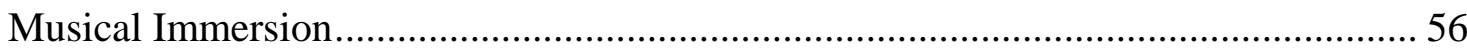

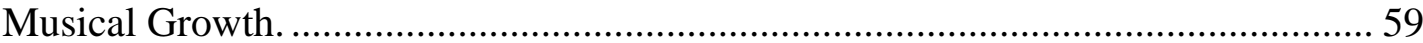

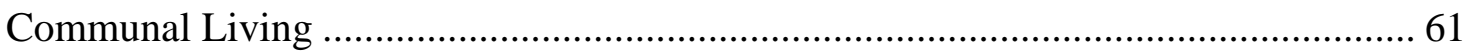

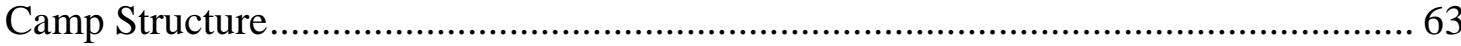

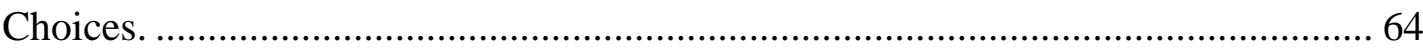

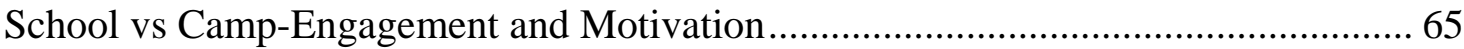

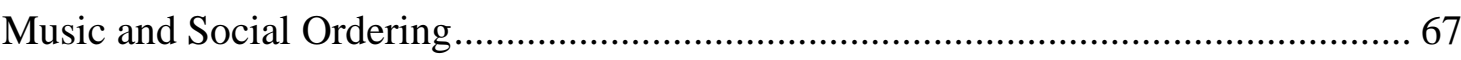

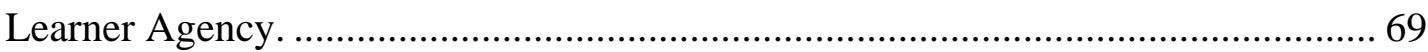

Collective Learner Agency-Peer Learning ........................................................ 70

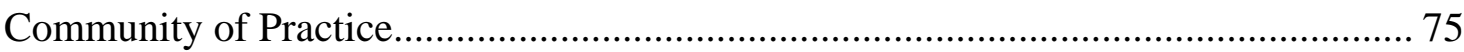

The Camp Bubble - A community Culture......................................................... 77

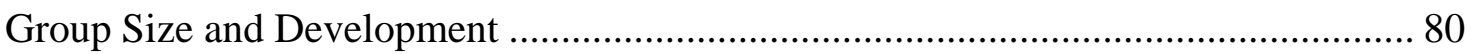

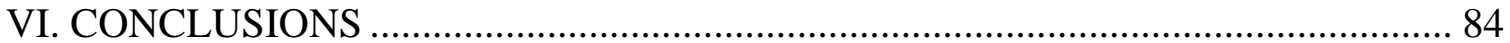

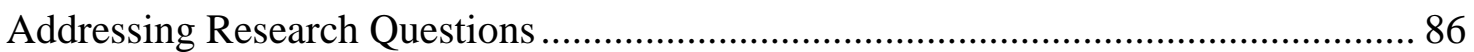

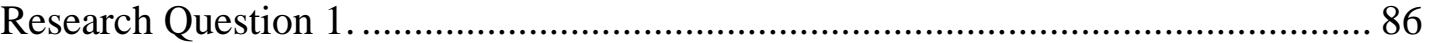

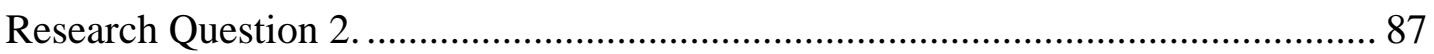

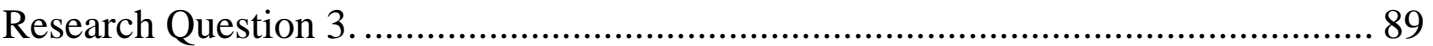

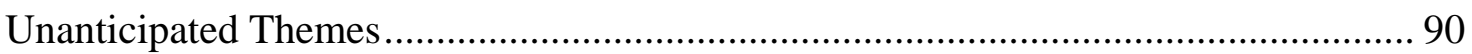

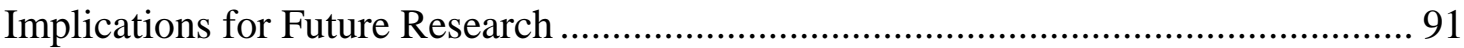

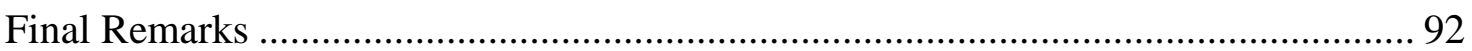

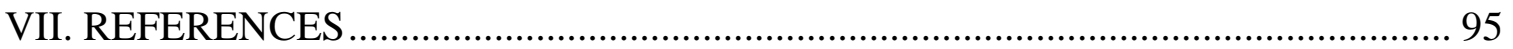

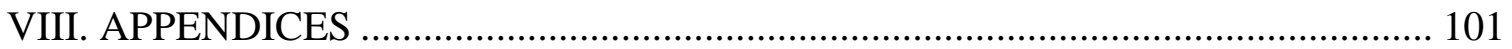




\section{INTRODUCTION}

For decades parents have been sending their children to overnight summer camps. The format extends for several weeks or even an entire summer and is a North American staple, mythologized in cartoons, novels, and movies. Initially, camps emerged out of dissatisfaction with traditional schooling and a desire for a well-rounded education that included physical exercise, mental challenges, teaching values, and social skills development (Thurber, Scanlin, Scheuler, \& Henderson, 2007). Historically, summer camps provided a haven and resource for parents, serving as a place where their children could be sent during summer school break. Their continued popularity with parents and youths owes much to the need for exciting and motivating ways to get youth away from urban areas, immersing them in a natural environment where they can interact with both peers and mentors within a holistic learning experience that contrasts markedly to the academic and social experiences that occur during the other months of the year.

Summer camps in North America can be traced back to 1861 and the founding of The Gunnery Camp in Connecticut (see http://www.acacamp.org/anniversary/timeline/). Since then, camps have flourished and evolved over time into the wide variety of programs available today. From their early years to the present, camps proved a sensible option for working families in need of day care for their children during the summer. In addition to providing a safe and supervised environment, camps have also created an enriching and exhilarating experience for the campers.

The first significant summer music camp materialized in 1928 with the inception of “The National Orchestra Camp” in Interlochen, Michigan. This camp emerged from the perceived need to continue instruction and performance in the summer for a select 
group of high school orchestra players. The young musicians who participated played not only for prominent and supportive music educators, but also for the President Woodrow Wilson (Brandt, 1988). Joseph E. Maddy, professor of music at University of Michigan, led this visionary endeavor. Along with the support of the National Bureau for the Advancement of Music and summer camp pioneer Willis Pennington, "The National Orchestra Camp” got off the ground in 1928 with minimal funding and low enrollment. Plodding forward with recruitment and external support, the camp saw its enrollment double the following summer (Brandt, 1988). Surviving the Great Depression by exponentially increased endowments, the extensive music camp and organization that exists today is now simply called Interlochen.

Interlochen's successful camp model spread rapidly across the country, sparking the emergence of other overnight music camps and summer music programs based at universities and preparatory schools. One significant such camp was the International Music Camp (IMC). Founded in 1956 by Dr. Merton Utgaard, at the Peace Gardens of North Dakota, IMC is recognized as one of the first prominent camps to intentionally recruit both domestic and foreign students (Brandt, 1988; Hall, 2000). With the institution of music and arts camps, the 1950s witnessed the establishment of camps with even further specializations, offering focused studies and activities such as baton twirling, pipe organ, dancing, and jazz (Brandt, 1988). The last segment of the camp model to spring up was the creation of music intensive summer camp programs based at colleges, universities, and preparatory schools. These programs thrive today at various universities and music conservatories, such as Florida State University, Penn State University, and Berklee College of Music. 
A distinction must be made between summer music "camp" and summer music “program.” The intensive music programs that emerged in the way of Interlochen’s establishment exhibit many similarities to traditional overnight summer music camps, but they also bear distinct traits. In these college camp programs, students can either camp by day or overnight, living in college dorm rooms and attending classes in university facilities. Berklee College of Music defines its program as a place where "the focus is on you and what you want to say and do through your music, in an environment that is all about performance” (http://www.berklee.edu/instrumental-programs). While musical immersion and performance are certainly a significant aspect of traditional overnight summer music camps such as Encore Coda, there are many other traits that define the environment. These include socialization, creative exploration, and the components associated with holistic learning within a community of practice. One camp director at Encore Coda contextualizes the difference, stating:

There are all kinds of programs out there in the world that call themselves a camp. But when you talk to camp directors who are running residential programs in the middle of the woods, like Encore/Coda, I think they would be very strongly biased towards the term 'camp' being used to define that sort of program, as opposed to a one week program at a private school or a college. (J. Saltman, personal communication, August 6, 2013)

The traditional overnight summer music camp, as explored in this study, is modeled after Interlochen. This model is one in which music students live together in cabins in the woods, typically in close proximity to a lake; living and learning takes place in a natural outdoor setting. This distinctive setting characterizes the traditional overnight summer camp that has evolved into the complex learning environments envisioned by music educators such as Dr. Utgaard. The present study is limited to the exploration of one 
traditional overnight music camp environment; Camp Encore Coda, characterized by a holistic education rooted in multidimensional growth and the construct of a community of practice as the theoretical framework.

\section{Problem}

Summer camp provides a significant part of many youth's overall education, expanding their regular school curricula by providing opportunities to try new ways of learning. Hampton (2008) reports the unique opportunities youth may experience at camp, such as the high level and frequency of personal contact with faculty, as well as the opportunity to perform weekly in front of large, critical audiences, all of which are rare in their high schools, according to the students interviewed. The youth developmental outcomes described in the summer camp research literature has provided educators with anecdotal information on the impact these camps have on their attendees, however, research exploring the impact of established music camps within the United States is almost nonexistent (Thurber et al., 2007; Richards, 2007; Westervelt et al., 1998).

The scarcity of music education research attempting to explore and provide an initial evaluation of the learning and social potential of music camps is restricted, investigating particular aspects of the specific camps as opposed to detailed explorations of the whole environments and the intricacies within. Music camp studies have investigated how camp affects college choices (Hampton, 2008), how educators view the camp (Belin, 2009), or how the camp impacts one particular pedagogical concept (Diaz and Silveira, 2012). The lack of research uncovering ways youth develop in this particular context leaves many unturned stones for music educators and parents seeking to make forward progress in the field of music education. The present study follows 
Hall's (2000) recommendation that further research should be pursued, with greater focus on particular aspects of a music camp, a comparison of multiple camps, or the exploration of another unique music camp environment in the United States.

\section{Research Questions}

In order to explore the issues articulated above, the following research questions will guide this study:

1. In what ways, if any, does overnight music camp facilitate musical and personal growth?

2. In what ways, if any, does the learning experience at overnight music camp differ from that of the traditional classroom?

3. In what ways, if any, do participating youth perceive and articulate these possible differences?

\section{Purpose}

This exploratory investigation serves to provide a better understanding of the complex learning and interactive dynamics within one music camp environment. It attempts to uncover the possible unique ways the camp environment may impact the social and musical developments of its participating youth. In surveying both the social and musical aspects of camp, the aim is to uncover what, if anything, makes it a unique learning environment, and in which ways the camp facilitates growth elements that may differ from those available within the traditional classroom. Managan (1989) defines a traditional classroom as one using a predetermined, academically based curriculum. Typically, this curriculum is explicit, "manifest in publicly stated goals of education" 
(Joseph, 2011, p. 50). Replicating effective,engaging, and student centered curricular approaches is a significant component and anticipated outcomeof this study.

\section{Significance}

The exploratory nature of this study may pique interest and open doorways for future research on the music camp environment. According to Hampton (2008), exploratory studies open pathways and are conducted to satisfy the curiosity of the researcher. This study represents another lens for seeking out ways to create optimal learning conditions, expanding on recent research such as Diaz and Silveira's (2012) study of flow among participants at a summer music camp. The current exploration is particularly significant for music educators who strive to understand the role of context and pedagogical orientation upon musical growth.

Furthermore, findings on the possible positive effects this environment has on young artists may lead to greater advocacy for such camps; potentially increasing further research. One of the anticipated outcomes of this study would be to develop awareness on the part of more educators and parents as to the positive impact these summer music camps can have on students. This increased awareness of benefits could create further opportunities for both youth and teachers to participate in the overnight music camp experience.

According to Richards (2007), training teachers to be prepared to work with diverse populations is an existing challenge in our educational system. Overnight summer music camps not only serve their participating youth, but also the teachers, and preservice teachers engaged within, creating a breeding and training ground for young as well as experienced teachers. The significance of the time teachers spend teaching music 
in the summer months sharpening their skills in different educational settings directly impacts, in a positive way, the quality of our entire music education system (Belin, 2009). Additional studies, such as the investigation of the staff experience would be a significant outcome of this initial survey, providing further insight into the staff roles, exposing opportunities available for continuing education and employment.

\section{Role of Researcher}

During my time on staff at Camp Encore Coda in 2012, I was able to gather a sense of the camp's culture, its educational methods, and the overall feeling of the environment. This past experience laid the groundwork for my interest in this inquiry and established my credibility with the directors, faculty and staff, and the returning campers. My data collection was unobtrusive to the daily flow and routine of the camp staff and campers and I was present during camp activities solely as an observer. Interaction with participants of the study included casual conversations around camp, interviews, and focus groups without any direct involvement in any camp activities whatsoever.

\section{Ethics}

As per standard procedures with any study involving human subjects, I adhered to a strict compliance of ethical considerations which included obtaining written consent from all participants and from parents of participants under the age of 18. All participants were made aware of the study's purpose and significance. Before consenting, participants were informed that their involvement in the study was fully voluntary, permitting them the option to withdrawal at any time. I paid special consideration and attention to the comfort of the participants, ensuring that they felt no pressure whatsoever to respond to questions they might feel uncomfortable answering. Lastly, all information collected 
pertaining to participants and their responses were kept anonymous, maintaining ethical considerations for confidentiality.

\section{Methods}

This investigation was established as a case study bound by Camp Encore Coda alone. I collected data from August 4-10 2013, the last week of camp’s final session for 2013. Case studies are intensive, bounded examinations of the unique qualities present within a unit or environment of study, bound by time and activity (Creswell, 2003; Stake, 2000). Merriam (1998) suggests a case study is useful in constructing a holistic description of an environment, rich with experiences, events, interactions, and characteristics, but bound by a finite time for observations and limited number of interviewees. A case study design may be pursued because it is intrinsically interesting, allowing the researcher to discover the intricacies that construct the particular case.

Data collection consisted of observations, interviews, surveys, and one focus group. Informal observations were conducted daily, including various activities ranging from ensemble rehearsal, concerts, recreational time, meal times, and others, allowing me to examine the intricacies of camp life. Formal observation sessions of the campers were conducted daily, focusing on both social and musical aspects. As part of my triangulation of data, I administered a survey to participants on the first and last day of each session. Returning campers were distinguished from first time campers through the survey data. The surveys used both a Likert-like rating scale and open and close ended questions addressing areas of musicality as well as specific social skills such as self-confidence or responsibility. Fourteen study participants were interviewed, including veteran music faculty, veteran counselors, camp directors, and upper camp campers (ages13+). Lastly, I 
conducted one focus group session, comprised of four upper campers who had also participated in the surveys.

I analyzed the data by separating it into themes and sub themes. This process is known as axial coding whose aim is to make conceptual connections between a category and its subcategories findings. Throughout the analytical process the researcher records analytical and self-reflective memos, thereby enriching the process, documenting thoughts and making implicit ideas explicit (Strauss and Corbin, 1998). According to Denzin and Lincoln (1994), the purpose of this process is to build theoretical frameworks to explain the collected data. 


\section{LITERATURE REVIEW}

This investigation of one overnight summer camp seeks to uncover the various aspects of the camp environment, deciphering what possible impacts such camp experience may have on the attending youth. A review of existing literature fosters a clearer understanding of this study's context, while linking previous scholastic knowledge to the current findings. This section reviews prior research, beginning with an exploration of what the camp environment is and what makes it distinct. Following this background is the contextualization of key concepts framing the study, namely, the following: peer interactions and relationships, an examination of the traditional classroom, community of practice, alternative learning opportunities, and holistic education. Together they construct a general framework upon which summer camp as a phenomenon can be investigated.

\section{The Camp Environment}

The overnight camp environment derives its uniqueness from many factors including: the natural setting, the staff, the clearly defined daily camper schedule, the interaction between youth living together, the interaction of youth with staff, and the overall structure and learning philosophy that the camp promotes. It is within the strategically organized interweaving of these components that the distinct environment is formed. Campers within this setting not only receive specialized instruction and daily intensive practice, but they also get to “just be kids” for several weeks. Here, youth are away from home, sleeping in a cabin with several others similar in age, enjoying campfires at nighttime, eating in a dining hall as a group, engaging in silly camp games, 
making new friendships, and at the same time developing and discovering who they are, as individuals.

The natural setting in the "great outdoors" is a major component of the camp experience and a clear element drawing youth to the programs. Camp is a living and breathing experience where children and youth interact in a safe environment (American Camp Association, 2006). This environment is set up in a fashion that facilitates numerous opportunities to make personal decisions and engage in meaningful experiences that become lifetime memories. One longtime faculty member and former camper from the International Music Camp recalls his first experience at camp when he met his trumpet instructor under a tree for a private lesson (Hall, 2000), painting a vivid example of camp learning in an authentic context.

Camp has proven to make long-term impacts on participants. This is seen in the return of campers for multiple summers, the return of staff for multiple summers, and the transfer of their knowledge from camp experiences into the world. Thurber et al. (2007) assert that developmental gains observed in camp are for the most part maintained post camp. Several of these gains include "important life skills, such as thoughtful decision making, wise use of resources, responsible citizenship, acceptance of differences, respectful treatment of others, and positive leadership” (p. 243).

In an effort to cater to as many different camper interests as possible, many camps pride themselves on offering an extensive array of activities to choose from. Among these are the many specialty camps offering instruction focused on development of specific skills such as sports, the arts, or academia. Both general camps and focused specialty camps provide a variety of activities ranging from soccer, horseback riding, swimming, 
arts and crafts, field trips, and evening camp-fire time. It is this combination of activities integrated with focused instruction and marked social experiences that make for unforgettable summers, and consequently, bring campers back, time and again.

\section{Peer Interactions and Relationships}

A great deal of research has been performed on peer interactions and relationships given their importance as major facets of life that directly connect to learning inside and outside of the classroom (Barstead, 2012; Rubin et al., 1998; Thurber et al., 2007). In order to clearly understand how the complex socialization of youth occurs, it is useful to delineate the distinction between the two terms, specifically how the occurrence of multiple interactions create relationships. This division of terms facilitates a conceptualization of how they both impact the various aspects of life, particularly learning. Rubin et al. (1998) explain interaction as "the social exchange of some duration between two individuals" (p. 576). A classic illustration of this is when two people are engaging in a conversation. The specific behaviors in various interactions can vary greatly, due to many factors such as short and long term personal goals, understanding of the partner's thoughts and feelings, physical setting, presence of others, and the operative local customs for responding (Rubin et al., 1998). As for the other connected term of study, relationships "refer to the meanings, expectations, and emotions that derive from the succession of interactions between two individuals known to each other” (p. 577). Relationships are a product of multiple interactions. Indeed, Rubin et al. (1998) suggest that "the degree of closeness of a relationship is determined by such qualities as the frequency and strength of influence, the diversity of influence across different behaviors, and the length of time the relationship has endured" (p. 577). 
Dyadic relationships between individuals share features with many larger social organizations such as schools, teams, or camps (Rubin et al, 1998). One key characteristic found in all of these contexts is a shared sense of belonging. The sense of belonging to a community, such as a school, is a basic psychological need (Baumeister et al., 1995). Crucially important for youth, various research points to poor achievement among students resulting from the lack of sense of belonging. Furthermore, research also points out that having a sense of belonging in a community positively impacts motivation and overall academic performance among students (Baumeister et al., 1995; Cueto et al., 2010; Goodenow, 1993; Joven, 2006). According to Barstead (2012), a sense of belonging provides many developmental benefits including improved mood, enhanced reasoning, better self-regulation, and increased self-esteem. A sense of belonging yields outcomes encompassing three human areas: cognitive, behavioral, and emotional outcomes (Cueto et al., 2010).

Another feature of personal relationships is the creation of a shared culture. Rubin et al. (1998) describe this shared culture as one that includes:

Normative expectations regarding appropriate activities, patterns of communication and revelation, relations to external persons and organizations, and so on. It also includes private terms, or neologisms, for shared concerns or common activities, and rituals, or 'dyadic traditions', arising from the routinization of recurrent dyadic activities such as meeting at the same place after school, flipping a special coin to resolve a dispute, or engaging in an exclusive "buddyshake" to mark a joint promise or planned behavior. (p. 577)

This culture tends to transpire among groups that have a common interest and often some degree of reciprocal influence over each other (Rubin et al., 1998). An array of properties emerged from groups in this context. One of these qualities particularly relevant to the 
setting of overnight summer camp is cohesiveness. Cohesiveness influences youths' sense of belonging (Rubin, 1998) and within a community of practice tends to be very strong (as shown in this study's data). Barstead (2012), a former camp program director with over ten years of experience in the field, asserts that camps are able to foster this sense of belonging by removing artificial barriers of social interaction and promoting a culture of togetherness within a community.

Rubin and his co-researchers concluded that research has not consistently demonstrated a clear understanding of the ways in which interactions, relationships, and groups are influenced by environmental conditions. Conclusions drawn from a single level of analysis can be limited, particularly with diverse cultural perceptions and a huge pool of variables in every case. This lack of conclusiveness makes the study of Camp Encore Coda a significant addition to the existing body of research, as the study illuminates the way these social processes function in a unique environment and how they are perceived by both the youth and the adults. These perceptions come from people who are also well accustomed to interactions linked to educational or academic association occurring within a traditional classroom environment.

\section{Traditional Classroom}

In the United States most people experience the bulk of their formal learning and training in the familiar "traditional" classroom setting. The notion of "traditional" is often challenging to define and identify due to the significant variance in existing educational settings that could appear to fit this label. Several distinct characterizing traits, however, help distinguish this particular learning environment. The traditional classroom for the purpose of this study can be defined as the classroom environment prevalent in most 
public schools in the United States. This context can be characterized by a walled classroom where learning approaches commonly, but not always, involve direct teacher led instruction. Commonly, the traditional classroom is based on a predetermined, academically based curriculum (Managan, 1989) mandating that core standards be met and assessed by both standardized and non-standardized tests. In the traditional classroom students are taught with students of similar age, and often ability, led by a unified imposed curriculum. In this configuration peer interaction is often limited due to the lecture- based model of instruction (not always but generally speaking) and separation of content level based on assumptions regarding developmental capacity according to age. Another characteristic of the traditional classroom is the nature of student -teacher relationships. In most schools they are formal, where students refer to teacher by last name and the relationship typically ends when the class concludes at the end of the period. In the essay entitled “The Kind of Schools We Need”, Eisner (2002) notes and warns against many of the treacherous approaches occurring in education, routines that have become standard elements of the traditional classroom. He accomplishes this by stating that the classroom and its pedagogical approach should:

1) Be places in which teachers have access to other teachers so that they have the opportunity to create that kind of supportive and educative community that culminates in higher [levels of] education. (p. 577)

2) Recognize that the most important forms of learning are those that students know how to use outside of school, not just inside school. (p. 581)

3) Stop reinforcing our students' lust for point accumulation. Point accumulation is not an educational aim. (p. 581)

Within the traditional classroom educators and students face several challenges and limitations. The first is a limitation on peer interaction, a natural aspect of life that 
facilitates increased opportunities for peer teaching and learning. According to Boud et al. (1999), "peer teaching commonly involves advanced students in the same class, or those in later years, taking on limited aspects of a teacher's instructional or pedagogical role” (p. 414). The separation of students by grade level and tendency toward large group instruction has become a root cause for this limitation.

Within predetermined curriculums, substantial limitations pertaining to space for creativity are frequently observed. According to Hickey and Webster (2001) creativity can be characterized by risk taking, attraction to ambiguity, and open mindedness. They assert that for this to be possible one must possess "enabling skills and an enabling environment to support the process” (p. 20). A supportive environment is one in which divergent and convergent thinking meet, both becoming essential components of the nurturing of creative practices (Webster, 1990). Creating this situation remains an ongoing challenge for educators to implement as fostering such an environment is often influenced by many external factors including financial support, family conditions, media, societal expectations, and peer pressure (Webster, 1990).

Teachers and students must adapt to and battle several other ongoing challenges in the traditional classroom as education evolves. Some of these challenges I have observed first hand including: lack of interest from the students, students showing signs of being tired and hungry at relatively early hours, and an overall lack of enthusiasm for the information being taught. Many of these challenges can be overcome in certain settings, particularly those considered to be a community of practice, such as summer camp. 


\section{Community of Practice}

Extensive educational research continues to be devoted to context, uncovering the ways in which different settings affect learning. Communities of practice are defined by Richards (2007) as being "social units that have a common purpose,” where "members interact regularly, share common beliefs and vocabulary, and learn from one another as they engage in mutual activities”(p. 40). Smith (2003) argues that a community of practice is a place where groups can tackle problems and share knowledge outside of traditional structural boundaries. Therefore, a community of practice is a unique environment that can exist both within the school classroom as well as outside it, in settings such as social organizations, sports teams, or camps.

Research by Jean Lave and Etienne Wenger (1991) has connected community of practice to the pedagogical model of situated learning. The authors proposed that learning involves a process of engagement in an environment where people generate meaning by functioning as full participants in the world. In their inquiries, they ask "what kinds of social engagements provide the proper context for learning to take place” (Lave and Wenger, 1991, p. 14). Situated Learning is further contextualized by Smith (2005) as a process where learners participate in structured frameworks where "learning is, thus, not seen as the acquisition of knowledge by individuals so much as a process of social participation,” a community of dependent experience “where the nature of the situation impacts significantly on the process” (p. 3).

The social engagements queried by Lave and Wenger within a community of practice are exactly what one experiences in an overnight summer camps. Existing literature characterizes overnight summer music camps as communities of practice. This 
characteristic environment and its experience are described by a camper in Hamptons's (2008) music camp study stating: "We all have a common interest. We all just kind of relate. I have met one of my best friends here actually” (p. 78).

Such a community presents the opportunity for flourishing relationships between mentors and mentees. The connection that campers have with the staff has been described as "a spirit of unity", one that is "fostered by the faculty and staff and is emulated by the students in both their artistic and personal endeavors during their camp experience" (in Hall, 2000, p. 281). This unity not only occurs between staff and camper but between staff and other staff. The common interest among all the staff and the embraced possibility to learn from each other is an integral part of summer camp and a part of teacher education that is commonly ignored (Richards, 2007). The intricacy of these relationships in such an environment can be seen as both a product of unconventional learning opportunities as well as the pieces making up these alternative learning experiences.

\section{Alternative Learning Opportunities}

The concept of alternative learning maintains a position in the academic world that co exists and challenges more "traditional" curriculums. In many countries music is learned in a semi-structured fashion, one with a daily routine of music making activities led by older community members and passed on to the younger ones. In this situation, learning outcomes arise from the master and apprentice model where older more experienced members within the community help the younger ones, acting as musical models that the younger musicians can talk to, listen to, watch, imitate, and join with in playing (Green, 2008). This example shares the structure and role distinctions with which 
most people of western educational tradition are familiar with, but lacks the predetermined curriculum that exists in the majority of traditional western classrooms.

In some settings, the community of learners embraces more of an informal learning model, one that affects the way skills and knowledge are transmitted. In this model, the musical authority no longer rests in the hands of adult figures within the community, but rather gives power to the learners. This is observed, for example, in small ensembles such as a jazz combo or rock/pop band, where peer learning within the group prevails, as the members work together discovering different ways to play and create music, a process that begins and ends where the members mutually feel is appropriate (Green, 2008). This contrasts the formal learning model typically governing school curriculums, where "its emphasis, very often, [is] on reproduction more than creativity... following a planned progression from simple to complex" (Green, 2008, p. 10). It is also common for young musicians to engage in what Green (2008) refers to as solitary learning, where students are alone, outside of any formal learning structures, discovering music on their own. Both of these models can be considered informal learning, characterized by a holistically approached process void of an adult teacher figure; where the music is chosen by the student(s) and often learned aurally.

Despite the distinct differences of formal and informal learning practices, there exist settings where these models merge creating unique environments that embrace both the structure of the traditional classroom as well as the more holistic approach experienced during informal circumstances. Westervelt, Johnson and Murrill (1998) uncover this dualism in the study of Camp Glencoe, where they found the camp offered many features not experienced by most children during the school year. Some of these 
included a structured and balanced day where they received one-on-one instruction targeted to their needs, recreational activities that range from challenging to purely fun, creative arts, and group living with peers and young adult counselors. According to “Innovations”, a youth summer experience improvement project commissioned by the American Camp Association (2006), camper retention rests largely with "the capacity of the camp environment to interest and challenge campers...doing different things than they do at home” (p. 10). Along with all the pleasurable activities, camp also implements for the youth responsibilities associated with structured daily routine. It is common for campers to fall into a daily regimen of straightening up living areas, cleaning up after meal times, and arriving on time to their activities based on a schedule they have created according to their interests. This variety of "daily life" activities, dependent on both self and peers, fosters the development of teamwork, problem solving, responsibility, and interrelationship skills (Kelley and Juchniewicz, 2009).

Exposing campers to a plethora of new learning opportunities combined with structured daily living void of modern technology encourages a more holistic experience that includes activities such as contact with nature and more interpersonal involvement (Westervelt et al., 1998). This kind of holistic living structure in a natural environment is fertile ground for youth development such as building self-confidence, one of many positive growth areas they can experience away at camp (Bhattacharyya et al., 2011). 


\section{Holistic Education}

Holistic education stems out of the worldview perspective of holism, the understanding that the whole is always better than the summation of its parts; that the whole of any context is more meaningful and complex than specific fragments making up the whole. This wholeness is said to be inherent in any phenomena and has sparked the learning approach of a holistic education, where emphasis on developing the whole brain

or the whole person is paramount. The holistic paradigm does not replace prior world and pedagogical views. Rather, it serves as a critique and possible learning approach to be incorporated within existing systems of education (Miller, 1992). This is precisely what was seen in the exploration of Camp Encore Coda, an integration of holistic learning with direct and grounded teaching approaches as found in many traditional school classrooms, but established within the context of a unique environment, defined by the existing physical setting and community of practice.

According to Miller (1992), humanistic beliefs are a defining characteristic of holistic educational traditions. Humanistic beliefs assert that, “education must give students the opportunity and skills to integrate academic learning with personal meaning and purpose” (p. 62). This approach does not aim to mold students, but rather, embraces a profound concern for students as individuals, their self- development, interpersonal skills, preparedness for future roles in society, and joy in learning. Carl Rodgers, a leading figure in the human potential movement, exhibited great influence on humanistic education, expressing the principal themes of holistic learning as an approach where the whole person is embraced: their feelings, emotions, creativity; within an emotionally supportive environment that encourages exploration and self-discovery (in Miller, 1992). 
Another significant component to holistic education is a focus on experiential learning. Dewey (1966) demarcates the importance maintaining "an ounce of experience is better than a ton of theory simply because it is only in experience that any theory has vital and verifiable significance” (p. 144). In conjunction with Dewey’s support of learning through experience, Miller (1992) describes the nature of experience as being "intentional and always reflect[ing] a meaningful connection between the individual and the world” (p. 100). Miller goes as far as to argue that this is indeed the role of education, to enable persons to look critically at the world, potentially relinquishing past traditions and beliefs in response to their new experiences. Overnight summer camps seem to find close kinship to this kind of experiential idea of learning, one where children and youth are given the space to think critically about the world around them, building meaning in all that they learn through both academic and social endeavors.

Several recent studies have examined the impact of holistic education in various contexts. Tirri (2011) investigated the perspectives of Finnish teachers and students in two different schools after an HBSC (Health Behavior in School-aged Children) study comparing student school satisfaction of 35 countries showed Finland coming last with the lowest percentage. Despite Finland's particularly high test scores, the low satisfaction was based on pervasive negative views of Finland's school atmosphere by the students, who felt the "opportunity to participate and influence their pedagogical environment was minimal” (Tirri, 2011, p. 159). This lack of holism in a national school system revered as highly successful makes Tirri’s study of two different holistic based schools in Finland particularly significant from a comparative perspective. Both schools in this study 
provide a holistic based learning approach with specialized math curriculums for students who are particularly interested in math, in addition to other major academic subjects.

The overall findings showed that both schools exemplified a community of practice; where the students' specific academic interest in math was stressed in conjunction with a highly supportive social environment that was crucial to the ethos of the school. This important intersection of holistic learning within the context of a community of practice is supported by interview data collected in this study, as well as past camp studies that show how the link between multidimensional growth in a supportive community setting of like-minded peers is a distinct foundation to the success of these programs (Hall, 2000; Hampton, 2008; Kelly and Juchniewicz, 2009; Thurber et al., 2007). In Tirri’s (2001) study, teachers were interviewed and asked to describe their current and past teaching experiences, sharing their motives for choosing to teach at their current school. In both schools, the teachers emphasized the importance of community to their students' personal growth. One teacher describes this significance in an interview stating:

The students learn social skills here and find a community with like-minded people. Many of our students have been bullied in their former schools, but here they see that they can be themselves in a friendly environment and learn scientific thinking and form a scientific worldview without losing their personalities. (Female boarding school teacher)

Interviews with students found overall satisfaction in both of these two schools studied. According to one student:

I am the intellectual type, not the athletic type, and enjoy the company of similar friends. In secondary school, the special curriculum in mathematics attracted people like me, and I have been friends with them. (Boy from regular school) 
Approximately 50\% of the students interviewed also expressed that in their previous schools they experienced bullying and social isolation. These new schools gave them a place where they could fit in, allowing them to excel socially and academically at the same time.

In order to fully understand how holistic learning is approached and paint a picture of how it has become significant to education, it is important to investigate its role in various contexts. Modell and his co-researchers (2009) investigated how creating a supportive learning community within a class of medical school students would aid in the development of all aspects of the students' learning. In 2009, they conducted two pilot studies:

To test the hypothesis that providing students with a participatory music experience may help to promote a holistic learning environment by helping them restore a sense of balance to their emotional well-being as well as reinforce a sense of community in the classroom. (p. 37)

The researchers assert that "in the context of a holistic learning environment, helping the learner to learn includes creating an environment where factors that are not directly related to the discipline but that may impact learning are recognized” (p. 37). The researchers created this environment by introducing a guest musician to the medical class. The musician spent time in the class facilitating group singing as well as organizing group discussions where she would ask students about their life paths, approaches to school, as well as issues raised by the students about life experiences. This occurred during periods of high stress for the students. The discussions served to assist the learners in discovering how to deal with different everyday life factors, many of which must be dealt with in the context of a high pressure and high stress medical career. 
It is important to note cautionary elements of holistic education, as those suggested by Modell et al. (2009). The creation of such an environment that adopts a more holistic education has challenges. A central challenge is the reconceptualization of the instructor's role by broadening his/her interpretation of what it means to help the learner learn. In a holistic context this means "creating an environment where factors that are not directly related to the discipline but that may impact learning are recognized and steps are taken to help the learner deal with these factors” (Modell et al., 2009, p. 37). Another obstacle of marked significance is the history many learning environments have of being extremely competitive, as commonly seen in the field of medicine. Often in these competitive environments students are accustomed to teacher-centered learning where the focus is short term retention of information conveyed by the teacher. Because these students are adapted to content-oriented learning rather than process-oriented learning, "many students are reluctant to participate in community-based, processoriented learning aimed at promoting meaningful learning” (Modell et al., 2009, p. 37).

Based on the positive results from the students and the professional review of the design of these training programs, it was discovered that "programs whose design is often consistent with common classroom practice, are often much less effective than they could be” (p. 45). The study concluded that:

The concept of a holistic learning environment is based on the recognition that students have both academic and nonacademic stressors that can negatively impact their learning. If we are to help the learner to learn, we must help students minimize the influence of these stressors on their learning. (p. 45)

The central conclusion from Tirri's (2001) research confirms similar ideals as those of Modell et al. (2009), with emphasis on nurturing the whole of each student to give them 
the tools they need to become independent thinkers capable of developing their own worldviews in addition to the mastery of specific subjects.

\section{Multidimensional Growth}

Research consistently points to holistic educational environments as the breeding grounds for multidimensional growth in youth. Thurber et al. (2007) describe camp as a place where youth benefit from a holistic experience including community living, exercise, mental challenges, and social skills improvement such as making friends from different cultures than their own. Their study entitled Youth Development Outcomes of the Camp Experience: Evidence for Multidimensional Growth affirms the concept by characterizing camps as:

Ideal for positive youth development: They are intrinsically motivating, "structured voluntary activities" with ample opportunity to take initiative, take risks, and develop mastery. This theoretical framework clearly predicts that children at high-quality camps should evidence multidimensional growth. (Thurber et al., 2007, p. 243)

The areas of growth potential for youth at camp can be directly related to the ideologies of the camp and its staff. Belin (2000) describes the teachers at IMC as “focused on the whole student - each student"s behavior, socialization, emotional wellbeing, and accomplishment in music” (p. 54). Gillard, Roark, Ramsey, Nyaga and Bialeschki (2011) elaborate on this socialization stating “camp is one setting for experiential education that holds the potential to foster positive relationships and competence-building opportunities that offer experiences for youth to initiate and engage in behaviors that aid in the transition to adulthood” (p. 89). The musical accomplishment aspect is quite distinct in specialty music camps where there is dramatic evidence for gains in the participating youth’s performance skills (Belin, 2000). The specialized 
instruction and overall immersion in music for extended periods of time, with other campers pursuing a similar interest, tends to nurture this progress. This combination of guidance in social skills as well as musical skills lays the framework for the concept of multidimensional growth in this study, a term synonymous with holistic learning.

Another impact of camp on attending campers is long term retention of information. According the American Camp Association (2006), youth attending summer camps have proven to retain new information learned in school more efficiently than those who have not attended. This has become a recent topic of inquiry in the field of education. One such study by Diaz and Silveira (2012) examines the concepts of flow, "a state in which individuals report enhanced levels of effortless concentration and enjoyment as a product of intense engagement on a given task” (p. 2). Their study seeks to identify how flow is achieved in the camp setting so that it can be applied to the school classroom and other learning environments, attempting to improve overall learning efficiency and retention. Further longitudinal studies on the long term learning and information retention impacts of overnight music camps would be worth investigating in the future.

\section{Summary}

This review of the literature delineates various educational contexts, exploring the different ways in which their various elements and overall orientation impact learning outcomes. The concepts explored in the literature review include peer interactions and relationships, traditional classroom distinctions, community of practice, informal learning, holistic education, and multidimensional growth. These larger constructs were chosen to frame the research of one overnight summer music camp in order to better 
understand the environment's complexities, and help the researcher better understand and consequently frame the investigation. The camp exhibits many traits central to these constructs, making it important to have a relational perspective of various learning models and contexts so that one can fully grasp the significance of the present study. 


\section{METHODOLOGY}

\section{Rationale for Qualitative Design}

According to Denzin and Lincoln (2003) qualitative research “consists of a set of interpretive, material practices that make the world visible...done by turning the world into a series of representations including field notes, interviews, conversations, photographs, recordings, and memos to the self” (p. 4). They further suggest that in qualitative research the researcher tries to make sense of the meanings people develop within a natural setting. Qualitative studies are often rooted in participatory and exploratory perspectives, foundations that should reveal the essential elements of the environment being investigated as well as the impact on its participants. Creswell (2003) describes qualitative research as "research that is emergent rather than tightly prefigured" (p. 181) and element that was clearly present in the process that led to this investigation, where the flexibility of the qualitative approach allowed for a natural emergence of themes and meaningful representations unfolded during the current research study.

In a qualitative study, it is necessary for the researcher to use a wide range of interconnected interpretive practices, as each practice makes the world visible in a different way. These practices make up Denzin and Lincoln’s (2003) description of the researcher's role as that of “a bricoleur, a maker of quilts, one who pieces together a set of representations that are fitted to the specifics of a complex situation” (p. 5). These practices can be summed up as building constructs by looking at data through a variety of lenses: methodological, theoretical, interpretive, and narrative. As a result, "this process creates and brings psychological and emotional unity to an interpretive experience” (p. 
7), therefore aiding in the construction of complex arguments that represent the depth and richness desired in qualitative studies.

\section{Case Study}

A case study was used in this research project as the method of inquiry, organizing the exploration of one particular camp environment and its population, namely, Camp Encore Coda. According to Creswell (2003), in a case study "the researcher explores in depth a program, an event, an activity, a process, or one or more individuals” (p. 15). Furthermore, case studies are intensive, bounded examinations of the unique qualities present within a unit or environment of study, bound by time and activity (Creswell, 2003; Stake, 2000). It is possible for case studies to be written with different motives, ranging from "simple presentation of individual cases or the desire to arrive at broad generalizations based on case study evidence” (Yin, 2003, p. 15). Depending on the desired motives and data collection approaches used in the study, a case study can be qualitative, quantitative, or a mix of both. According to Yin (2003), a case study is an appropriate design for their research if it logically links the data to be collected to the initial questions of study. Merriam (1998) suggests a case study is useful in constructing a holistic description of an environment, rich with experiences, events, interactions, and characteristics, but bound by a finite time for observations and a limitation on the number of participants interviewed. The characteristic trait of a 'bound study' is fundamental to case study research and is considered the appropriate context for an exploration that aims to uncover qualitative data on one particular phenomenon.

The bounded system in this study was chosen for its uniqueness and appeal, a reason that is supported by Merriam (1988) who argues that the aim is to find a case that 
"it is itself intrinsically interesting, and one would study it to achieve as full an understanding of the phenomenon as possible” (p.10). The goal in this sort of study is “insight, discovery, and interpretation, rather than hypothesis testing” (Merriam, 1988, p.10).

According to Yin (1984), case studies also serve to elicit understanding where little research has been explored previously, and in turn create an outlet for further in depth studies. This model is non experimental and descriptive, where the researcher explores the case in its natural state without the intention or ability to manipulate the environment, the subjects, and potential causes for behavior (Merriam, 1988). Yin (1984) asserts that in this kind of design it is impossible to separate the variables of the system from their context, a situation perfect for holistic description and explanation.

While an abundant amount of qualitative research has been done on traditional summer camps and various specialty camps, little investigation has been pursued that focuses on the environment of overnight music camps. The qualitative case study is well suited for this research as it provides an in depth view of this phenomena from two distinct participant pools, students and staff, and four different data collection methods, namely, surveys, interviews, focus groups, and observations.

\section{Role of the Researcher}

According to Creswell (2003), “qualitative researchers look for involvement of their participants in data collection and seek to build rapport and credibility with the individuals in the study” (p. 181). During my time on staff at Camp Encore Coda in 2012, I was able to get a sense of the camp’s culture, its educational methods, and the overall feeling of the environment. This past experience has laid the groundwork for my interest 
in this inquiry and established my credibility with the directors, the faculty and staff, and the returning campers. The data collection process was unobtrusive to the daily flow and routine of the camp staff and campers. As the researcher for this study, I was present during camp activities solely as an observer. Interaction with participants of the study included casual conversations around camp, interviews, and focus groups. Direct involvement in any camp activities did not occur. It is important to note however, that I have participated in camps previously, working as an instructor for Camp Encore Coda in 2012 and as a counselor for another overnight music camp in 2008.

\section{Ethics}

A significant aspect to the protocol of this investigation was a strict adherence to ethical concerns pertaining to the participants in the study. According to Merriam (1988), whenever the researcher possesses a degree of influence or control over study participants, there is a danger of exploitation and thus a need for guidelines and regulations. In compliance with guidelines set up by government and institutional authorities, I sought full IRB approval which was confirmed prior to any data collection. After approval by the IRB, I obtained signed consent forms of all participants, including parental consent if the participant was a minor, which was the majority of campers.

One ethical consideration for the present study included paying careful attention to the participants' freedom of choice and thought. This was adhered to by refraining from pressuring individuals to participate and not compelling participants to answer any questions they felt uncomfortable addressing. Avoidance of coercive activity is an essential part of the researcher's role, something he/she must be conscious of from the conception of the problem to the dissemination of the findings (Merriam, 1988). Another 
major facet of this protocol included confidentiality of the participants' personal information and participation. Protecting the anonymity of participants is extremely crucial, so as not to risk offending or implicating them in any post research investigations that could potentially result based on information divulged in the findings (Merriam, 1988).

\section{Context and Description}

The camp used in this investigation was established in the early 1950's and continues to thrive today. Encore Coda attracts youth from various parts of the United States and internationally. The directors hire music faculty with the highest levels of music degrees and experience; and on a regular basis bring in nationally known and recognized artists to work with and perform for the young musicians at camp. The camp characteristics place it as a reliable model for a study on the summer camp environment, its population, and a close look at the unique musical and social benefits a specialty camp of this nature can provide to its attending youth.

The camp is co-educational containing a balance between female and male campers, with numbers at approximately 140.The camp population count also includes a faculty of experienced music educators with masters and doctorate degrees, counselors of the college age from various parts of the world, and a staff specializing in other crafts

necessary for the various activities and overall operation of the camp. Campers primarily come from the north eastern part of the United States (85\%); however, there is a small percent of the population coming from other parts of the US (10\%) as well as other countries (5\%). Due to the highly specialized instruction provided at Encore Coda, the overall socioeconomic status (SES) tends to be on the higher side, but this is not to say 
there are not youth from families of lower SES, since the camp offers scholarship opportunities to make camp accessible to families who could not otherwise afford the full cost of tuition. The camp is comprised of two sessions, the first being 3.5 weeks and the second being 3 weeks. Families can choose to send their sons and daughters for only one or both sessions. I collected data on site throughout the final week of session two, August 4-10 2013.

\section{Data Collection}

In qualitative research data is presented though words and descriptions rather than numbers. This is accomplished by entering into the environment of study or phenomenon under investigation; in an attempt to become close both physically and psychologically to that which is being studied. Through this close proximity and the engagement in a variety of data gathering methods, a qualitative researcher is able to put together a well justified picture detailing aspects of the phenomenon uncovered during the investigation. For this type of data to be considered valid, the researcher must use multiple entry points into the environment of study. This use of data triangulation is employed in the present study, pulling facts, perspectives, opinions, and interactions from several sources, namely observations, surveys, interviews, and focus groups (Merriam, 1988).

Observations. There are several reasons for collecting observations, including identification of routines among participants, seeing things first-hand, and gaining insight from behavioral situations that otherwise might not be revealed in other data collection methods. According to Merriam (1998), these first-hand “as it is happening” observations enable the researcher to "use his or her own knowledge and expertise in interpreting what 
is being observed, rather than relying on once-removed accounts from interviews” (p. 89).

Deciding what to observe and determining whether it justifies the conceptual framework of the study is a key concern. Merriam states that "observation is the best technique to use when an activity, or event, can be observed first hand, when a fresh perspective is desired, or when participants are not willing to discuss the topic under study” (p. 89). Observation is further justified as a research tool when it "(1) serves a formulated research purpose, (2) is planned deliberately, (3) is recorded systematically, and, (4) is subjected to checks and controls on validity and reliability” (Kidder, 1981, p. 264).

In the present study, observations were conducted daily, encompassing all facets of camp life from meal times to structured and unstructured musical and non-musical activities. Upon arrival to Encore Coda, I received a detailed schedule of camp activities so that observation sessions could be planned out for the week. According to Creswell (2003), observations can be structured using prior questions the researcher wants to inquire, or semi-structured where there are no expectations and merely what is observed is what is recorded. I executed both approaches to observations during my time at camp. The observations were recorded through note taking during the actual observations. The notes were separated into two categories, subjective and objective, enabling the sights and sounds of the moment to be captured as well as the interpretations of the researcher. The objective notes were taken solely in real time while subjective notes were chronicled during both the actual observations and after. According to Merriam (1998), more complete recordings of observations make decoding and analysis easier as it helps paint a 
fuller, more complete description. Supporting this notion, I recorded several stream of conscious reflection videos at several points during the week of data collection.

Surveys. According to Babbie (1990), surveys are an apt choice when making generalizations from a sample of the population, allowing inferences to be made about characteristics, attitudes, and trends of the entire population. Particularly for the purposes of this study, surveys established the meaningful variation within a population rather than establishing frequencies or means (Jansen, 2010). While surveys have the potential to collect data in a variety of ways, in the field of education they usually contain selfcompletion questionnaires and attitude scales. Cohen, Manion, and Morrison (2011) state that this model tends to be used as a tool for:

Gathering large-scale data from as representative a sample population as possible, in order to say with a measure of statistical confidence that certain observed characteristics occur with a degree of regularity, or that certain factors cluster together, correlate with each other, or that they change over time. (p. 257)

For this study, I administered two separate surveys, each on the first and last day of the session respectively (see appendix B). Since I did not arrive to camp until the second week, the camp faculty administered the initial survey. I administered the final survey, permitting participants to take the survey with them and return it completed before leaving camp. Surveys delineated various statistical information regarding the camper population, i.e. which participants are returning to camp and which are first time campers, as well as more in depth information from open ended questions. All components of the survey addressed areas of musicality as well as specific social skills such as self- confidence or responsibility to name a few. These surveys represented an 
exploratory model, one which according to Cohen et al. (2011) is helpful in acquiring factual information, data on attitudes, opinions, behaviors, and experiences.

The survey design administered offers many advantages to this particular case study. One primary advantage, characteristic of many surveys (Cohen at al., 2011), is that it had an economy of design, one in which the youth who took the survey could easily understand and complete with thoughtful responses in a short time of no more than 20-30 minutes. The rapid turn-around time in data collection fit well with the time restrictions of this study. The camp staff and I administered the surveys in a paper format, omitting any unnecessary extra expenses, making the survey very cost efficient. Lastly, the conglomeration of question styles gave the participants space to express themselves in a variety of ways while breaking up the flow of the survey so that attention to detail and overall interest was maintained. Cohen et al. (2011) recommend organizing a survey from general to specific in terms of content, allowing the survey to flow in well-defined stages. Further, they assert that several items or questions for each component issue be presented throughout the study as it supports the validity of the data and "does justice to the allaround nature of the topic” (p. 259). The strength of this model was confirmed through the detailed responses received and wealth of thematic patterns that emerged.

Interviews. They serve the function of eliciting information in a person- to person encounter, one that mirrors a regular conversation but with purposeful intent. The aim of the researcher is to get into the mind of another person, uncovering thoughts, perceptions, experiences, and any meanings they may attach to the world. The interview process allows for data gathering in a way that observations cannot, extracting perceptions from the subject pertaining to both present and past events (Merriam, 1988). 
In the present study I conducted a total of 14 interviews with veteran staff members and campers (ages 13+) (see appendix C). The reason for delimiting the camper interviews to upper camp is supported by Chichucki’s (2007) assertion that young adolescence is when youth begin exhibiting independent and critical thought. I conducted nine staff interviews comprised of music faculty, bunk counselors, and directors, as well as five camper interviews. Interviews were semi- structured, based on observations and preplanned questions, but adjusted to specific individuals and circumstances as I saw fit. According to Seidman (1991), "the more one wishes to acquire unique, non-standardized, information about how individuals view the world, the more one veers towards qualitative, open-ended, unstructured interviewing” (p. 412).

By nature, a qualitative study aims to use open ended questions so that the participants can express their views with as little leading and bias from the researcher as possible (Creswell, 2003). To this end, a significant component to uncovering participant perceptions and experiences was the engagement of youth through casual conversations at various points each day. While not officially documented, these conversations also contributed to an overall understanding of the camp experience, evidenced in the voice and video memos made throughout the week.

Focus Group. The last component of data collection was the recording of one focus group session. I facilitated this session with a set of pre-planned discussion questions. Focus groups can be defined as a carefully planned series of discussions designed to obtain perceptions on a defined area of interest in a permissive, nonthreatening environment (Krueger \& Casey, 2009). These groups usually contain 4-8 participants and are valuable for discovering individuals' opinions as well as interactive 
opinions, or the way the participants react to each other in regards to a specific discussion. Due to the nature of the group, focus groups are less useful for gaining information sensitive topics, but rather, unveil more opinions, emotional reactions, attitudes, and beliefs towards topics the participants feel comfortable discussing openly (Morgan, 1996). The focus group serves as a secondary function, with the surveys providing preliminary information and the focus group digging deeper into the responses that emerged out of the surveys. According to Morgan (1996), "what makes the discussion in focus groups more than the sum of separate individual interviews is the fact that the participants both query each other and explain themselves to each other” (p. 139). Such interaction offers valuable data on the extent of consensus and diversity among the participants.

\section{Validity}

The method of data gathering used follows primary principles of qualitative research, collecting data from multiple sources in order to achieve triangulation. Creswell (2003) states that triangulation attempts to build a coherent justification for themes based on the evidence. When all the sources of evidence are reviewed and analyzed together, the findings are based on a convergence of data, rather than on a single source. Using converging lines of inquiry makes conclusions more convincing and ultimately more accurate (Yin, 2003).

Similarly, "the potential problems of construct validity can be addressed because the multiple sources of evidence essentially provide multiple measures of the same phenomenon” (Yin, 2003, p. 99). Construct validity is a way of justifying the framework and components planned for the study, checking that they make sense for the particular 
situation under investigation. According to Yin (2003), construct validity establishes “correct operational measures for the concepts being studied” (p. 34), rationalizing the method as appropriate to the study and justified through triangulation.

\section{Coding and Data Analysis}

Upon completion of data collection, the process of coding commenced with a detailed review of each piece of data obtained while on site at camp. This included listening to and transcribing interviews and one focus group, reflecting on observation notes, reading survey responses, and listening to my personal stream of conscious videos that were recorded sporadically throughout the data collection period. The information uncovered in this data was decoded using axial coding. The aim of axial coding is to make conceptual connections between a category and its subcategories findings. Throughout the analytical process the researcher makes analytical and self-reflective memos, thereby enriching the process, documenting thoughts and making implicit ideas explicit (Strauss and Corbin, 1998). According to Denzin and Lincoln (1994), the purpose of this process is to build theoretical frameworks that explain the collected data.

After decoding, the data was linked to literature review and managed in order to create a complex interpretive network regarding the case. Through the use of a narrative, the findings of the study are presented in a story-like manner that shares the researcher's perceptions of the phenomenon through detailed descriptions of events and interactions that took place (Merriam, 1998). 


\section{Limitations and De-limitations}

This study is delimited by the examination of only one summer music camp. Camp Encore Coda has a well-established program of many years with a very clear, systematic, and structural approach to instruction and daily life. Although it shares these characteristics with other camps, Encore Coda has unique aspects, such as its smaller size. While the size provides a distinct and complex environment for investigation, the study is limited to the impact possibilities of only Encore Coda and its camper population of approximately 140 individuals. Other well established overnight music camps exist in the United States, supporting enormous populations that triple or even quadruple Encore Coda in size. The similarities and contrasts of camp impact on youth from different size music camps will not be identified in this study but could be a significant comparative investigation in the future.

As a past faculty member and advocate of music camps there is some bias on my part as the researcher. A similar situation also exists with the returning faculty and staff that were interviewed. To limit bias as much as possible, questions in the interviews were primarily open-ended and constructed to ensure minimal leading of the participants’ responses. Because qualitative research is fundamentally interpretive, it is difficult, if not impossible, for the researcher to escape personal interpretation during data collection and analysis (Creswell, 2003). For this reason, questions were framed very carefully.

Another limitation of the study is the time frame by which the data collection was bound to. My time on site at Camp Encore Coda was limited to six days, August 4-10 2013. This restricted observations, interviews, and focus groups to the final week of the second summer session. Further longitudinal studies may permit for greater depth of data 
and overall analysis, particularly regarding the camp's long term impact on attending youth. 


\section{DISCUSSION}

Upon arrival at Camp Encore Coda the feeling of entering an isolated world in the woods, away from the familiarities of modern society, becomes immediately apparent. The use of vehicles on camp grounds is limited to a few camp staff members and the occasional visitor. Scattered around camp are various brown, well preserved, wooden structures, accessible through a connection of dirt paths. The wooden structures where most of the teaching and activities take place have electricity, but no heating or air conditioning. These structures include small practice huts/lesson studios, large rehearsal and performance halls, health lodge, dining hall, bathroom facilities, and cabins for living.

At the center of camp is the administration building. Hardly what one imagines when one conjures up “administration building,” the space resembles more a lodge one might find at a camp ground or ski resort in the United States. The building is in three levels. On the ground floor there is an ensemble rehearsal room beside a staff lounge. The second level is approached by symmetrical wood staircases that meet on a deck. Immediately evident are two piano studios, each on one side of the final staircase that leads to the third level. The deck wraps around to the back of the building, with ping pong tables on both sides of the deck. Towards the back of the second floor there is a music library and room that is used for various rehearsals and music classes. Interestingly, the ping pong tables have become a significant addition to this building, serving as a popular place for campers, faculty, staff, and of course one of the camp directors (an avid ping pong player as well as professional musician) to meet sporadically during the day and night. Ping Pong at Encore Coda provides participants a break from 
the music, and exemplifies the kind of space that creates a social environment where campers, staff, and administration come together for fun.

The top floor is a place where the directors and assisting administrative staff work. Various music faculty and cabin staff can also be found, as they come and go handling different situations and work related tasks. Isolated, and far from city noises and distractions, this is Camp Encore Coda, a premier summer music camp.

Camp Encore Coda is located in the woods of Sweden, Maine, off the beaten path and approximately fifteen minutes by car to the nearest town. The camp property is situated on the edge of Stearns Pond, one of many fresh bodies of water in the area. The property is engulfed by a forest containing numerous species of trees, plant life, and the occasional roaming creatures typically found living in this woodland environment. One can best understand the size of the camp grounds in terms of traveling by foot, taking approximately no more than ten minutes to move from one end of camp to the other. Along this walk of mostly dirt paths that roll up and down depending on the terrain, the occasional area of open grass area, the recreation/sports field, or the well-maintained green area in front of the administration building, interrupts the ebb and flow of movement.

\section{Cabin Living}

At Encore Coda campers are divided into upper and lower camp, terminology that distinguishes between campers finishing grades three through six for lower camp and grades seven through twelve for upper camp. Campers of similar age are grouped together into their respective cabins for the summer. The cabin is a home base and central hub for every camper. This is the place where many initial and often close and long 
lasting friendships occur. This is where campers may relax and socialize, where they practice alone or with peers, and where they sleep. The cabin represents a sub-structure of the larger camp community, a place where the youth tend to establish strong bonds and sense of belonging. As this is the campers' first introduction to the overnight camp experience, integration into the cabin may be easy for some, while for others, a challenge that must be overcome. Ultimately, what is observed here is a group of youth living together in a cabin, forming a cohesive unit built on the common interest of music.

The camper cabins are separated by sex, with male and female cabins located on opposite ends of the property. The lakefront and pine tree forest serves as an implicit central boundary between the two sides of camp. The lakefront is an important location at camp, serving the traditional function of boating and swimming, but also establishing a central location where campfires, cookouts, and activity nights all take place. Male and female camper interactions take place throughout the day, concluding at night when boys return to the boy's side of camp and girls return to their quarters. Cabins typically house 6-12 campers with bunk beds and cabin lighting for the accommodations. Restroom and bathing facilities are called "The Head," a wooden structure that serves the needs for a fixed set of cabins on each side of camp. These facilities are accessible by foot and require a flashlight when visiting after sunset.

Communal living plays an integral part to the overall experience at Camp Encore Coda. This element proves to be foundational to the camp experience, and is consistent with other overnight camp studies positing that living together with peers in an overnight camp environment fosters development of interpersonal skills, self -confidence, self concept, independence, problem solving, team work, and self-empowerment (American 
Camping Association, 2006; Bieleschki et al., 2002; Ozier, 2009; Thurber et al., 2007;

Westervelt et al., 1998). At Camp Encore Coda, communal living is a distinct way of life. In this living situation members interact as a unit, including socializing, cleaning, eating, and negotiating daily struggles that might arise.

\section{Structure}

At the onset of each three-week camp session, the campers design their own schedule, choosing the musical activities and recreational activities in which they want to participate. Creating schedules provides each camper with a daily structure to follow for the next three weeks. This initial step in the camp routine (in which students are given the opportunity to create their own customized daily schedule) lays the groundwork and sets the tone for a camp experience where student decision making aims to be an integral part of daily life. The importance of having choices is articulated by several campers, one saying "I am mostly looking forward to choosing my ensemble” (Camper Survey) and another stating "It's very good that campers can decide their classes. We can be much more independent” (Camper Survey). The sense of having responsibility for oneself, particularly via the opportunity to make personal curricular decisions, is very important at camp. This inclination toward more independent choices begins to emerge as a distinct trait of the camp experience, best expressed by a third camper who states that, "Without parents, you can make a lot more of your own choices” (Camper Interview).

While life at camp is filled with choices, structure and guidance are nevertheless a constant. During the three weeks a camper is away at camp they fall into a daily routine. A day in the life of a typical camper begins around 7:00 a.m. with wake up and cleanup. Campers are expected to rise at the same time so that everyone can prepare for the day, 
clean the cabin and bathroom facilities, and arrive on time together for breakfast by 7:30 a.m. Restroom facilities are fully maintained by the campers, each cabin takes turns assuming cleaning duty in the morning before breakfast. This cleaning duty is in addition to the daily expectation of group cabin cleanup. Cleanliness and responsibility for individual and group living areas is rigidly upheld at camp and publicly recognized when cabin clean up points are announced at chosen meal times: winners at the end of a session receive an ice cream party for placing first in the clean-up competition.

There are two scheduled times for each meal during the day, one for upper camp and one for lower. Meals are served in the dining hall, a building where campers sit by cabin and eat all of their meals together. The food is prepared onsite and consists of a variety of options, accommodating multiple diet preferences. There is no restriction for the camper on what they can eat and how much, however, counselors of younger campers do provide guidance when deemed necessary. Mealtimes at camp are a time for both food and mental nutrition. While campers eat they are also socializing, often discussing happenings from their various classes, rehearsals, and other activities. This time of coming together as a cabin, after parting ways to attend each ones' own unique schedule, represents a time of further communal bonding.

Between breakfast and lunch and lunch and dinner, campers attend their various classes, rehearsals, and activities. There is multitude of activities from which to choose, ensuring that every camper is accommodated and has a full schedule every day. The majority of this time is spent immersed in musical activities, with one to two hours a day available for non-musical activities. Several activities that comprise this schedule include large ensemble rehearsals, private lessons, musicianship classes, musical drama rehearsal, 
chamber group rehearsals, swimming/lessons, boating, field sports, arts and crafts, or model rocketry. Campers take responsibility for getting to their scheduled destinations, walking to their various activities without a chaperone. This extensive quantity of activities offered within the daily structural framework makes camp feel like a living and breathing experience, where staying busy and productive at a rapid and constant rate is the norm.

After a packed day of activities and dinner, campers have pre-planned social activities, concerts, and personal down time. Several special social events include casino/game night (fake money), Rhino Night (lip sync contest), and the camp dance. Campfire night is one particular activity that many campers really enjoy, encompassing a combination of music and skits (often on the comical side), performed by staff and campers.

\section{Concerts}

Performing for each other is a significant component of the camp experience, occurring in every context available within this environment. These include formally planned camper concerts in the concert halls, to outdoor rock/jazz festival, to community performances off location, to dining hall jam sessions at meal times, and very distinctly, the morning reveille played daily by a different solo trumpet player to wake up the entire camp.

Evening concerts are a staple of overnight music camp, occurring several times throughout any given session. The wide display of performances includes a variety of styles including classical, jazz, rock, pop, miscellaneous genres, and original compositions and arrangements. The performances are further distinguished by the 
ensemble sizes, ranging from solos and duets to chamber groups under ten members, to larger ensembles such as the full symphony orchestra encompassing approximately forty plus members. While these concerts mainly feature campers, one can also enjoy hearing the staff members perform at staff concerts.

Chamber groups are another space and outlet at camp where youth can be observed developing and discovering their musicianship. Whether in classical ensembles or more modern groups such as jazz combo, rock band, and various other smaller ensemble collaborations, the campers explore their musical interests in a place they feel they fit in, both musically and socially. For those students who choose to participate in classical chamber music, such as that traditionally played in a string quartet, there are many opportunities to play and receive professional coaching from the faculty. The classical groups also take advantage of time outside of class to rehearse on their own without instructors; a practice observed by all campers, regardless of instrument or musical style played. For those campers who feel more connected to jazz, rock and roll, or merely want to try a new style for the first time, there are combos formed by the camp that receive professional coaching during class periods like the classical counterparts. On several occasions campers with little to no improvisation or jazz experience, as well as beginners on their instruments, are seen participating within their small combo groups playing the heads and taking solos. A noteworthy trait of Encore Coda is the fact that campers are not accepted into camp based on ability. Students with varying levels of musical experience may attend, and it is strongly encouraged at camp to learn new instruments and styles one has not tried before. 
The jazz combos and rock bands at camp appear to have a coalescing effect on the lives of all campers. At the end of each session Camp Encore Coda has "Rock Fest", where all the camper rock bands and jazz combos play for each other outdoors in a section of forest near the lake. The environment is very laid back and enjoyable, a place where everyone supports each other, regardless of the quality of performance and wide range of ability levels. Campers have the opportunity to socialize with each other, eat snacks, and just hangout for the festival.

\section{The Environment}

An outside observer will certainly and easily notice the non-competitive environment fostered by this camp and remark on its possible supporting role in the openness toward the act of performing evident in most, if not all, campers. The support campers experience emanates from many sources, namely music teachers, counselors, and peers. Particularly apparent is peer support. Spanning all age ranges, this can be easily observed as eight year-old violinists watch and cheer for high school jazz band players and vice versa. The concerts prove to be very special times; a time of coming together and enjoying all the hard work everyone has been engaged in day after day. These concerts are more than just performances, but rather emotionally charged experiences where campers and staff can be seen laughing, cheering, relaxing, examining, bonding, and at times, shedding tears.

Time and Performance. Despite the non-competitive environment, there still exists a degree of tension and pressure that becomes apparent to any observer. This seems to come as a result of the three week time constraint every camper is held to. Camp sessions provide a relatively short and fixed amount of time to prepare for final concerts 
at the end of each camp session. Some campers experience performance anxiety, particularly those who have less previous performing experience during the school year. Several campers who expressed this feeling also demonstrated the motivation to get past it, accomplished through practice during the week and the constant support by the community around them. Overall, the pressure to learn music quickly and perform on a regular basis does not seem to be a burden to the campers, but rather, according to several campers, motivating and stimulating.

An observer will notice high levels of engagement and motivation from the campers, characteristics that manifest in a community of practice. This support occurs in a context where there is a clear delineation of ability levels, rates of progress, and musical goals. During both seemingly high and low pressure moments, it seems that students take pride and a strong sense of ownership in all of their endeavors. This can be witnessed during practices and performances as students take advantage of the space camp provides for them to be creative, composing and arranging works, or simply finding their own musical voice within the literature they are learning.

The notion of time plays an integral role in the camp experience and the overall impact it has on the attending youth. Students seem to find sufficient time for in depth encounters with their studies, able to focus on very specific skills such as jazz piano, ear training, or swimming, but also time for trying out new and/or less explored things. This could be anything from playing in a jazz or rock combo for the first time, to studying a secondary instrument, to singing and performing in the camp musical. Every day campers can be seen using time as they see fit. This includes diving into various musical and nonmusical endeavors during the pre-planned class periods or during various other times of 
the day where free time is provided for youth to engage in less structured activities of their choice. It is during many of these less structured times that a variety of musical happenings can be observed taking place. Students are sometimes alone, practicing musical parts for their lessons and ensembles at camp, composing, or simply just practicing technique on their primary or secondary instrument. On the other hand, these times also provide the space for campers to get together and conduct their own rehearsals where they might be either preparing for camp ensembles or engaging in sporadic jam sessions.

\section{Musical Immersion}

Camp Encore Coda possesses a uniquely distinct trait of fully immersing the students in a musical environment. A significant aspect of this immersion is the constant playing, listening, and exposure to music learning opportunities every camper receives. However, this musically immersive environment is not only defined by a place where the youth are making music all the time, as the social and recreational activities observed indicate this. The denotation of musical immersion is expressed by one camper who states "I like having the [practice] studios and walking by and everybody is just playing stuff” (Camper Interview), indicating that even as a passerby on foot, individuals at camp always have music around them. A visitor to camp would experience this as they walk to various locations, hearing the jazz band rehearsing off in the distance and simultaneously a vocal or string group rehearsing nearby under a tree.

Total musical immersion seems to be attributed to several factors. The most obvious is the overall common interest in music. This interest is combined with the desire to be surrounded by other likeminded individuals who also seek to expand their 
musicianship, particularly in ways they may not be able to do so the rest of the year. As one camper expressed, "the best thing about being here is that you are here with people who also like music" (Camper Interview).The other element of this musical immersion is the unique outdoor feature of camp. One camper describes this aspect saying, "You are never truly indoors here because there are screen doors and everything, you are never truly out of the fresh air into some stuffy room” (Camper Interview). Every rehearsal space at Camp Encore Coda has a degree of audibility and visibility from the outside in and vice versa. The environment is set up in a way that does not confine students indoors the way they would be in a traditional classroom. Consequently, if one is present on the camp property, they are constantly reminded it is a music camp by the always present musical happenings occurring throughout. Several faculty members at Camp Encore Coda contextualize this, describing life at camp as "living in the camp bubble" (Faculty/Staff Interviews). This "bubble” is not only constructed through the musical engagements at camp, but also the social interactions that emerge within the community of practice context.

\section{Social Interactions}

Social engagements of all sorts take place during camp in a diversity of situations. These interactions perform a catalyst role linking background experience to personal and communal ones, and thus facilitating bonding, sharing, and trust. One time during an outdoor dinner cookout a group of adolescent boys were observed sitting together at a picnic table. The table was comprised of several U.S. born youth and two campers from South American countries whose first language is Spanish. Several of the guys gathered there showed an interest in speaking Spanish, querying their peers about the language. 
Within minutes, a discussion unfolded, where they engaged in teaching each other various words in Spanish and English. This sparked the interest of another camper at the table who had studied French in school. The adolescents furthered the language learning to include both French and Spanish. This happening exemplified the plurality of social engagements at camp, where youth with different backgrounds and understandings come together through the common interest of music, subsequently sharing information and experiences.

Another unique type of interaction playing a major role at Camp Encore Coda is facilitated by the Camper-In-Transition, or CIT program. This program is characterized by older campers who have attended Encore Coda for several summers living in cabins with younger campers and working with them for several hours per day; a position that encourages them to embrace leadership roles. Campers are selected by the camp administration to participate in the program because of their maturity, good judgment, and interest in working with younger children. The CITs assist cabin counselors with daily routines of cabin living and various problems that arise, but also participate in the same array of camp activities as their peers. The camp directors describe their hopes for the participants of the CIT program:

To see each CIT develop his/her leadership skills through managing their daily interactions with the full range of other people in camp, learning to balance a class schedule with CIT responsibilities and handling new and sometimes challenging situations successfully. We hope that each of these experiences will help CITs develop a deeper understanding of human interactions and of how important thoughtful leadership can be in so many different settings and situations. (http://www.encore-coda.com)

Several counselors and faculty at Camp Encore Coda have moved up the ranks at camp, starting as campers, followed by involvement in the CIT program and eventually 
becoming counselors and/or music faculty. The CIT experience represents a significant transitional point in the lives of the participating campers, providing the space and context for them to take on greater responsibilities than they have in the past.

\section{Summary}

Camp Encore Coda provides a complex learning environment where campers with a common interest in music can come together for an extended period of time to learn music and have fun. Living together in cabins in the woods in a setting isolated from the familiar world provides many unique interactions, experiences, and challenges on a daily basis. The camp structure provides a safe and educational environment where campers have the opportunity to engage in an array of activities, musical ones at the forefront, but social and recreational ones equally important in their development towards adulthood. The non-competitive environment in conjunction peer and mentor support from living in a community fosters both the development of tightly bonded relationships as well as willingness to try new things. Campers expressed a satisfaction in living in a situation where they can make many personal and musical choices to guide their days. The camp environment provides attending youth a memorable learning experience that contrasts what they are familiar with at home, describing Encore Coda as their new "home away from home” (Camper Interviews), a place many return to for multiple summers. 


\section{DATA ANALYSIS}

The data obtained in this study uncovered an array of interconnected themes, which when brought together in the analysis below form a complex picture of the learning and social environment of one overnight summer music camp. Triangulation of data enabled access to the intricacies of camp through multiple entry points, uncovering how this complex environment is formed and how it functions as one particular facet of participants' musical education. Through the use of axial coding, data was separated into themes so that a framework of relationships could be constructed.

While the nature of qualitative analysis is interpretive, relying on researcherguided insight, the complex understandings presented below emerged from and are highly grounded in the data, and supported by the existing literature. Viewing the camp environment through various lenses has permitted the presentation of a critical and valid analysis that addresses the study's research questions. The questions explored here sought to uncover the impact overnight music camp might have on its attending youth, how it might compare to traditional school and music experiences therein, and how the youth perceive these possible differences. The following themes emerged from the data and are used below as a way to organized and make sense of the collected data.

\section{Musical Immersion}

When asked how attending camp impacts one’s overall musicianship, the dominating consensus from participants, yielding 100\% of survey and interview responses, was that music camp facilitates an accelerated rate of musical progress and growth in ways participants are not able to experience at home, at school, or during the other months of the year. In many cases both outcomes were noted. The $100 \%$ return of 
responses noting musical growth by participants matches expectations found in the literature. According to Hall (2000), participants who attended the International Music Camp evidenced rapid and dramatic improvement in their musicianship as a result of their experience. Camp Encore Coda has attracted participants for many years and has built a reputation as a musical learning center seen as worth-while by parents; parents who pay substantial sums of money so that their children may attend. Consequently, an emerging and recurring theme from the data is learning through musical immersion.

The data in this study seems to show that through a fully immersive musical environment the campers accelerate the expansion of their current abilities and develop new skills. This occurs through numerous opportunities to try new instruments, new musical styles, new approaches to learning, and musical situations that distinctively occur because of the environment established in an overnight summer music camp. Green (2008) refers to this experience as "enculturation, or immersion in the music and musical practices of one's environment, a fundamental factor that is common to all aspects of music learning, whether formal or informal” (p. 5). This process of enculturation is cited by several participants stating:

I'd like to think it's about really immersing yourself in the musical environment in a way that you can't do at home, knowing you don't have to worry about homework, you don't have your brothers and sisters at home [distracting you], and you really focus on playing your instrument or singing. (Faculty Interview)

Well...you are playing every single day which definitely helps. I think it's just the playing so much and with other kids here that really help musicianship. (Camper Interview)

There are a lot of rehearsals. The kids are in orchestra for two hours every day, and then most of them go off to chamber rehearsal, and then some of them do a 
secondary instrument, and they have their lessons, so you're looking at a pretty intensive schedule just there. (Faculty Interview)

There seems to be a distinct level of focus within this enculturated setting where a different kind of learning that goes beyond the effects of additional tie-on-task is established. Campers are not seen bothering or imposing on their peers’ practice sessions because the majority of youth attend camp for the same purpose, engaging in a wide range of musical activities. This environmental immersion appears significant and corroborates the findings in Diaz and Silveira's (2012) overnight music camp study, which exposed distinctly heightened levels of concentration during various camp activities, particularly the musical ones. This phenomenon is grounded in the concept of flow, which as they explain is "a state in which individuals report enhanced levels of effortless concentration and enjoyment as a product of intense engagement on a given task” (p. 2). The data in this study also point to the notion that marked increases in participants’ levels of flow at overnight music camp may be connected to the mutual understanding that they are in a learning community where everyone has personal goals they are trying to achieve. Apparent then is an interaction between immersion and flow, with one enforcing the other, as this one camper corroborates: "It's a place where music is what you do so you kind of decide early on I'm here to do music and I'm here to learn" (Camper Interview).

Another contextual element that emerges throughout the data as impactful on musical learning is the geographical isolation of camp. The data indicate that isolation from society in an outdoor/woodsy environment is an integral component of the camp experience, supporting the claim to heightened flow levels among participants. The 
connection to flow is evidenced in one camper's comment noting "The fun outdoor camp environment probably helps me stay concentrated” (Camper Interview). In this setting campers are removed from familiar technologies such as phone and computer. It is important to note, however, that the one technology permitted in addition to lighting is IPOD music. The overall view from participants was that the change in lifestyle created a positive hiatus from home life. One young female described this break as "refreshing” (Camper Interview).

The outdoor environment uniquely enables the creation of a "truly" musically immersive environment. At Encore Coda “you are never truly indoors” (Camper Interview). Sound proofing does not exist in the camp's wooden structures and many times doors and windows are left open. Even when not in a planned music activity such as ensemble rehearsals or lessons, there exists a constant sound of music in the background. Sometimes it is the light sound of a string group rehearsing outside in the field as you walk to lunch, and other times it might be a rock band jamming off in the distance.

Musical Growth. As part of the enculturation process campers improve their musicianship in ways both perceivable by themselves and other listeners, such as peers and camp staff and faculty. Two participants shared the impact camp has upon their musicianship stating:

This camp has given me [improved my] sight singing and is pretty close to making my pitch go from relative to perfect. (Camper Survey)

Whenever I'm here I get so much better at whatever I do, because camp forces you to get much better. I wouldn't be playing as much at school. (Camper Interview) 
Camp exists for these individuals as a completion to their music education, rounding out their musicianship through highly focused studies in multiple areas and offering numerous possibilities to explore areas of musicality unavailable to them elsewhere. The skill of sight reading emerged in the survey results with more than twenty percent of students mentioning marked personal improvement as a result of camp. The data seem to show that the skill of sight singing receives much emphasis in the camp curriculum, an area of musicianship many youth do not experience until college, should they pursue music.

The impact musical immersion appears as having other implications than the acquisition of musical skills. One camper shared her perception using a different lens than her peers above. When asked the question “what did you learn at camp?” she replied "how to be my own teacher" (Camper Survey). Her response substantiates the development of independence as a result of participating in overnight music camp. Further, it establishes the significance of the enculturation process at camp having both a behavioral as well as self-cognitive element. That is, that camp does not simply produce skilled musicians, but rather, individuals who discover how to prepare themselves for the world, becoming critical of the musical process, themselves, and the people and situations around them. According to Dewey (1987):

To prepare him [or her] for the future life means to give him command of himself; it means so to train him that he will have the full and ready use of all his capacities; that his eye and ear and hand may be tools ready to command, that his judgment may be capable of grasping the conditions under which it has to work, and the executive forces be trained to act economically and efficiently. (p. 78) 
The musical immersion model at camp acts as a foundation and springboard, facilitating the campers' discovery of their own capacities through instruction and exploratory experiences. This foundation is only one part of the camp context that contributes to youth development. As the data show, the strength and uniqueness of Encore Coda as an educational setting comes from a combination of elements.

\section{Communal Living}

The data in this study provide evidence of how communal living impacts participants, supporting the findings in existing camp literature. It is interesting to note the constant interaction among peers in close quarters. This condition creates an environment completely different from what the campers experience the other months of the year. Living in a cabin where peers are forced to interact, work together, share ideas, and struggle through daily challenges of living away from home. This confluence of events results in the formation of a cohesive unit where strong bonds of friendship are formed, one where tensions need to be managed and resolved. Several campers describe the impact of communal living thusly:

Well...you're forced to meet a whole bunch of people in your cabin the first day. It forces you to ask the small talk questions and the band pick up line "what instrument do you play”, and like how to talk. (Camper Interview)

The experience of living with people is even more bonding that going into a room with them for 6 hours in the day which is what it is at school. (Camper Interview)

How to live cohesively with 9 other girls, how to share space, how to be with them at all times. At home I have my own space, so here it's different. (Camper Interview)

The campers find themselves choosing to live in this environment, yet, forced to adapt to the internal/intrinsic elements of living closely with others. Communal living is 
far from what the youth are familiar with and creates a situation where they are in a sense engaging in risk-taking; a risk in living outside of their comfort zones, in an unfamiliar place lacking the amenities of home, and given the close proximity compelled to form new social relationships. Due to the fact that every camper manages the same general daily routine, facing similar challenges and changes in lifestyle where they must adjust, there is an inevitable series of mutual outcomes. These include, but are not limited to, the responsibilities associated with maintaining a clean cabin, arriving to meals on time, adapting to weather related events, and forming friendships in conditions that are often distinct from those experienced in their home environment. The overall nature of camp and communal living is described by one director stating:

This camp creates a unique situation where youth are learning to cooperate and collaborate with peers. At home they have the option to turn their backs on problems with peers simply by the ringing of the bell for the next class, by engaging with technology devices, and ultimately going home at the end of the day. At camp they are eating, cleaning, sleeping, practicing, creating, and playing with the same people all day and night. (Director Interview)

Within such a communal living situation campers experience a prolonged period social and life skill development. Some of the social and/or miscellaneous outcomes of this according to the campers include:

You gain experience on how to live with people (Camper Survey)

How to make my bed (Camper Survey)

I might be better at organizing things (Camper Survey)

Responsibility (Camper Survey)

Time Management (Camper Survey)

How to be a good friend (Camper Interview)

These are not unusually articulated within the literature, which addresses macro concepts such as independence, self-esteem, teamwork, and those mentioned earlier. Thurber et al. 
(2007) mention the building of life skills as a trait that characterizes the camp setting. The data presented above by the campers exemplifies a more specific set of life skills improved upon within the cabin living setting at Camp Encore Coda. Such skill acquisitions support the all-encompassing observation that camp personnel have created a rich and meaningful learning environment for the youth. According to Benedict (2009), "a rich educational environment is one that is student centered and serves the curricular function of shaping the ways in which students should and could be prepared to enter the world” (p. 148). It is evident from the data that such a communal living experience creates what might amount to a sociological space that optimizes the possibility for a transformative learning experience. One international student shared why she attended camp, stating "I wanted to immerse myself in American culture. We have this perception of summer camp in America being a big thing and it is, we don't have it at all in the UK and I just wanted to experience that” (Camper Interview). This camper found it easy to integrate in this new environment, despite being in a foreign country. Living in a communal situation where she could be immersed in both music and American culture, she realized Camp Encore Coda was a space apt for learning and growth on many levels.

\section{Camp Structure}

Just as in schools, camp maintains a clear degree of structure. According to one faculty member, "if you know something about child development, the kids this age may not act like it but they crave structure. They need structure” (Faculty Interview). According to Markam (2014), mindfully constructed structure and routine processes possesses many positive benefits to youth, including eliminating power struggles, facilitating cooperation, and taking charge of one's own activities. Some may think a 
clearly defined structure can become oppressive, but when well managed and carefully monitored, it has a positive effect. When starting from a pre-defined expectation of what needs to be accomplished every day, campers need less reminding and are provided the space for greater autonomy and empowerment. What might seem contradictory is based on an understanding that learning environments are complex and require a both/and, rather than either/or stance. Markam (2014) claims that "kids who feel more independent and in charge of themselves have less need to rebel and be oppositional” (para. 14). The empowerment associated with maintaining routine develops a sense of competence as the youth internalize the ability to structure their own lives, a skill they can take with them when they leave camp (Markam, 2014). The impact of developing competence and what it means to the campers is shared by a participant of the CIT program who stated: "I liked the feeling of being trusted and looked upto by the kids” (Camper Survey). This individual felt such satisfaction in fulfilling her obligations as a camper that she wanted to pursue a greater responsibility at camp by transitioning into a counselor for an upcoming summer.

Choices. Within the structure provided at camp, the youth are given the opportunity to make many choices of their own. These choices are wide ranging, mixing routine and complex processes, including but not limited to: food, activities, hygiene, musical repertoire, and musical decisions within ensembles. Several campers expressed how they felt in regards to the unique decision-making opportunities presented at camp: I love how everyone can choose a unique schedule. (Camper Survey) I am mostly looking forward to the ensemble of my choosing. (Camper Survey) 
In my combo I'm the only horn player and have the lead most of the time. It's fun to be the leader of the band, to have a little bit of control. (Camper Focus Group)

Maybe it's a little bit like what college would be like. Without parents, you can make a lot more of your own choices. (Camper Interview)

The data re-affirm that within a highly structured environment, there exists space for personal decision making. While the opinions above represent the majority of the camp population, it is important not to omit data that contradict these view-points. According to one camper:

I haven't had to choose anything for 3 weeks, you get it all handed to you. You don't have to think about anything, everything you do from the moment you wake up: you get given a job you have to do in the morning for cabin clean up, you go to breakfast and that's already chosen for you, than your activities start and they are organized for the entire day. (Camper Interview)

This particular camper was the only participant who perceived the camp structure to be a totally pre-determined context where personal decision-making is limited. Nonetheless, she has returned to camp for multiple summers. She shared many positive aspects of camp that seem to outweigh her feeling of restriction on choices including "[Camp is] a vacation but a constructive one” and [Camp is a] break from reality for a bit but also working constantly, learning new stuff and meeting new people”. The notion that overnight summer music camp is fun and feels like vacation, yet maintains the productive qualities of school, emerged in many interviews, surveys, and conversations around camp.

\section{School vs Camp-Engagement and Motivation}

School teachers also have the ability to create structured educational environments in their classrooms that engage students through meaningful experiences and a sense of belonging. According to Cueto et al. (2010) school engagement is “a 
construct that is closely related to sense of belonging” (p. 277). While overnight camp and the traditional school classroom maintain many differences, they are able to converge on pedagogical approach through the creation of a setting where students feel they belong. When this occurs, we can see in both contexts a positive outcome on student engagement and motivation.

Although one obvious possibility behind heightened student engagement and motivation at camp is the fact that mainly students with a niche interest in music attend, perhaps it is the context in which the learning occurs that drives their desire to stay busy.

One could conjecture that every child or adolescent loves music and that it is the way in which teachers present subject matter that positively or negatively impacts student engagement and motivation. According to Allsup and Benedict (2008):

We engineer programs that appeal to one kind of student; we offer one kind of music, we prescribe one method of teaching...the students who do not fit our mold are not our problem- we are satisfied with ten percent of the school population we enroll without considering that one hundred percent of our students enjoy music. (p. 166)

If one can agree that one hundred percent of students enjoy music, then one must inquire into what causes students to embrace learning opportunities as opposed to turning apathetic, a challenge every school-teacher faces at some point with various students in their classrooms.

Camp exhibits one answer to this question, offering a variety of musical styles, playing opportunities, and listening opportunities; opportunities that cannot be taken for granted in either public or private schools. One camper shares his dissatisfaction with the musical opportunities at school stating that even though there exists "a good sized music program at school with great people running it...I dislike that there aren’t a lot of places 
for a guitarist or drummer in it” (Camper Survey). This particular camper enjoys blues guitar and has found a way to explore his musical interests by playing with musicians around his town, as well as frequenting Camp Encore Coda. At Encore Coda he participates in rock bands, jazz big band, jazz combo, and solo and chamber guitar study, much of which includes composing and improvising.

According to the survey data not only is this level of diversity and focused experience rare within traditional schooling, camp participants also report what they perceive to be a significant lack of music in their schools. While some campers expressed deficiencies in the offerings of their school's program, approximately twenty five percent of survey responses indicated either thin programs at school or in some cases no existence of a school music program. Several of these responses included:

I don't do music at my small alternative school. (Camper Survey) There is no music in my school. (Camper Survey) There is barely any music in my school and I hate that. (Camper Survey) I'm homeschooled, but music at middle school is bad. (Camper Survey)

What becomes evident is that there exists a marked dissatisfaction with the musical learning opportunities available through their school education. Overnight music camp seems to provide supplementary musical opportunities for its participants, filling the gap in music education present outside of the camp context.

\section{Music and Social Ordering}

While many times campers take advantage of personal time to listen to their favorite music or something they may be studying, they also can be observed listening to and sharing music by playing it on a cabin speaker. This phenomenon represents a form of sharing whereby students have the opportunity to hear others' musical choices. Here 
music serves one of its many functions in everyday life, one that DeNora (2000) labels as social ordering. DeNora characterizes this function by emphasizing how "music may be employed, albeit at times unwittingly, as a means of organizing potentially disparate individuals such that their actions may appear to be intersubjective, mutually oriented, coordinated, entrained and aligned” (p.109). One example of this occurs during the morning, when it was common to hear distinct tunes blaring from a particular cabin as youth would go through their daily morning regiment of cleaning the cabin, the bathrooms, and preparing for the day. During one morning observation the following occurred:

The morning reveille woke everyone up fifteen minutes ago. The guys in the Stingrays cabin all look very busy. Two guys are sweeping the floor outside the cabin together, one guy has plastic gloves and appears to be heading to the bathroom for cleaning, and several others appear to be dressing and making their beds/cleaning the cabin. There is a pop music song blasting through the cabin windows and some of the guys seem to be dancing along as they work. It seems as if they are all enjoying these morning preparations. (Researcher Observation)

While many would simply characterize this encounter as "kids being kids", having fun to music in which they identify while performing the expected daily routine, I would argue that the precise and conscious manner in which choices were regularly made tell the story of a more impactful experience for the campers.

A further investigation into cabin life would uncover many of the facets making this event so significant. Some of these intricacies include: the execution of teamwork towards a common goal, the collaborative — and at times not so collaborative-process that occurs to determine who selects and plays the music in the morning, the distribution of jobs, and managing the challenges that arise during the process. These challenges may include uncooperative cabin members or discrepancies on how the morning routine 
should be completed. For most of the cabin members, sharing personal music choices during a communal morning regiment is something they do not experience at home or in school. While this was not originally anticipated, the issue of ordering social interaction with or through music is worth mentioning, and should be further explored as it might offer further insight into the social as well as subjective lives of youth interested in music. Furthermore, this kind of purposeful social ordering leads us to consider the notion of agency and the multifaceted ways in which it can be constructed by youth.

Learner Agency. According to DeNora (2000), the music "establish[es] a basis for collaborative action” (p. 111) and the data here collected support this notion, while pointing to the fact that music sharing represents an example of agency, both individual and collective. In this process, the youth are sharing their personal musical identities with their peers as well as developing new identities from the shared experiences of listening to a variety of music, all of which occurs in a collective context.

Another function of music also linked to agency is its role in regulating feeling, mood, concentration and energy level (DeNora, 2000). Wiggins (2011) defines agency as "the intentionality and control an individual feels she has over her own circumstances in a particular situation or at a given time in that situation” (p. 91). When campers' living quarters confine them to a tightly packed, one room cabin, with only basic furnishings and perhaps some posters on the walls, music is one of the few available materials for altering their encounters, taking control of the situation and dictating the factors mentioned before. Music in this context represents a medium for individual and collective agency, and ultimately personal empowerment. For an individual to engage in a meaningful music situation where they feel sufficiently empowered it is crucial they 
view themselves as an agent. This study's data show that group music listening facilitates agency, functioning as another facet of cabin bonding, highly influential and possibly the glue bringing everyone together. Lastly, it would not be inappropriate to conjecture that such fundamental engagement with agency might lead to or at least facilitate further and more complex or critical attempts toward agency, an extremely important element in the life of learners and of musicians.

Collective Learner Agency-Peer Learning. The notion of collective learner agency emerged as a central aspect to the camp environment. One of the Encore Coda directors describes the information sharing that occurs at camp stating:

There's the printed curriculum here, the stuff you can read about on the website we offer, but there's a whole second layer of unofficial curriculum going on, with the kids sharing music with each other, learning from each other. (Director Interview)

The data provide abundant examples of participants' comments regarding the extent to which peer learning occurs at camp. Based on observations, this camp fosters peer learning through the existing communal living situation of peers who share the same interest in music. When you place 6-8 musically motivated adolescents in a small cabin, removed from modern technology, each with a voice and an instrument beside their bed, the musical and social happenings that emerge create countless peer driven learning situations. In this space campers share stories, feelings and emotions, musical knowledge, and quite often collaborate in spontaneous music making. According to Rubin et al. (1998), "peer experiences have generally conceptualized the group as an important developmental context that shapes and supports the behaviors of its constituent members” (p. 579). 
During one observation session several adolescent males were seen sitting around a picnic table during an evening outdoor cookout diner. All were cabin mates, with several from the Unites States and two from South America. During this meal one US camper engaged in a conversation about Spanish language with his peers from Colombia, exchanging various words and expressions in English and Spanish. The entire table found the conversation interesting and input from several other members could be heard. Within a few minutes one camper stated he studied French in school and the conversation evolved into a multilingual learning experience for all present. Through collaborative information sharing processes, students are able to build upon the ideas and understandings of others so that they may extend their own ideas and understandings (Wiggins, 2011). This encounter represented a segment of spontaneous collective learning, initiated and embraced by the campers among themselves. The subject matter was not music related, but emerged from the social interactions of a tight knit group of cabin mates.

The experiences participants encounter living with peers in a cabin not only nurtures traits as responsibility, but also breeds spontaneous, exploratory learning from the social interactions that occur between cabin members. Many of the learning realities of camp are what Dewey (1938) describes as experiential learning, a series of unplanned events and interactions that emerge naturally from any given context. While the routines may be planned, the interactions and learning outcomes are not. In one instance, I walked into a boys’ cabin looking for a specific camper, and I witnessed two cabin mates who had free time that hour practicing their saxophones together. They were sharing music from their respective jazz combos, engaging in practice outside of planned rehearsals and 
practice hours. Ozier (2009) asserts that "just as children go about the activities of learning in classroom settings, similar skills are acquired through experiences in families, communities, and yes, even camps” (p. 4).

A major contrast emerged in the survey data regarding peer learning experiences at camp compared to at school. Over fifty percent of the surveys revealed campers encounter minimal or no peer learning experiences during the other months of the year when they are outside of the camp context. A lower percentage, approximately thirty-five percent, reported experiencing peer learning in school "somewhat", with only one camper reporting significant peer learning opportunities at their school.

Information sharing at camp among peers is also present in the various musical contexts. One place this was evident was in chamber rehearsals. During the focus group session several campers shared their excitement:

There was a rock study across from my cabin, I sat down and started playing the electric piano, and another kid form my cabin who was a drummer walked in and started playing drums with me, than a saxophonist from my cabin walked in and we were all jamming and it was really fun. (Camper Focus Group)

Another member of the focus group jumped in the conversation sharing a memory from that same instance, "We ended up practicing multiple times without a coach, and we traded fours [rotating four bar solos in a jazz context] and it was awesome” (Camper Focus Group). During this storytelling of memories, all the campers seemed to exhibit great excitement about the spontaneity of this musical happening, as well as a sense of pride in rehearsing together and performing at the camper concert, all without the assistance of a camp faculty ensemble coach. When students are not conscious of the teaching, but rather learning takes place naturally through watching and imitation, this is 
what Green (2008) refers to as 'group learning'. Green also characterizes another form of learning as 'peer directed learning', where teaching is explicitly peer driven and there exists a conscious sharing of knowledge. In both cases, "the absence of adult supervision or guidance are of great importance” (Green, 2008, p.7). At camp, learning music without adult guidance uncovered how deeply students feel about being empowered through learning with peers. This particular experience exemplified one of many student driven musical collaborations at Camp Encore Coda.

Camper concerts are one camp activity that exemplifies information sharing on a large scale. One observation uncovered this phenomenon very distinctly:

This is the second camper concert of the session. Everyone (campers of all ages, staff, faculty, administration) is assembled in Saltman Hall. Campers are sitting by cabin, younger campers up front and the older teenagers towards the back. Every counselor is here with their cabin and all the music faculty appear to be present as well sitting in the back. It seems not one person at camp is missing from this event. I notice something distinct about the hall (besides its totally rustic wood construction), hanging from the ceiling are large flags, approximately 20 or so representing various countries from around the world. Perhaps this is a symbol of universal coming together in a place of music. During the camper performances everybody seems engaged. Regardless of how "good" or "bad" a performance is, everyone is showing support to the performers through silence during the performance and often loud and animated applause and cheers before and after. (Researcher Observation)

These camper concerts are a really central part to camp life. The coming together of all community members after long days filled with activities makes these events very special. Everyone at camp is equally tired and everyone has really worked hard towards their various goals. During one concert observation the emotional impact of the event was exposed at one of its peaks when "during the campers performance the room remained silent and fixed on the young boy singing with his guitar, but more noticeably several girls from the upper camp cabins began weeping with tears running down their faces, 
clearly moved by the performance” (Researcher Observation). The concerts transcend age, sex, gender, ethnicity, musical preferences, and ability level. They are the culmination of many hours of hard work, creating a warm environment where sharing music without judgment, without competition, and with full community support defines the experience.

One central element to Camp Encore Coda existing as part of the camp culture is the creation of a non-competitive musical environment. Two different faculty members confirm this stating "it’s not a super competitive atmosphere" (Faculty Interview) and “there's not so much competition here, in the way that it's not about who is the best player at camp, it’s much more a social event to come here” (Faculty Interview). The impact this has on learning as perceived by the youth is expressed by one camper who articulates that "the non-competitive environment encourages us to teach each other" (Camper survey). It can be postulated that when competition is omitted from a learning environment, knowledge sharing amongst the group tends to occur more frequently, as observed at Encore Coda. The non-competitive environment in this camp creates a place where youth feel comfortable exchanging knowledge, through performing and peer learning situations. Looking at the substantial musical developments uncovered earlier in the data, one can infer that the non-competitive atmosphere has a positive impact on the musical progress of youth who attend camp.

Regardless of the overall perceptions above, it is important also to note that tensions or alternative views exist. One camper, for example, shared a contrasting pedagogical approach at her at school stating, "I have outstanding instructors who prepare us very well for competitions” (Student Survey). While this study does not attend 
to the question of competitiveness directly and therefore further investigation of this theme is not possible, what can be determined from the data is that the learning experience at overnight music camp impacts youth differently from their traditional school classrooms in regards to socialization and the musical outcomes associated with living and learning in an environment defined by music and relationships. The significance of the non-competitive model is delineated by Ruben et al. (1998) asserting that "noncompetitive activities facilitate socializing and the development of relationships” (p. 592-3). It becomes clear from this statement and the socialization observed at camp that the non-competitive atmosphere upheld at Encore Coda is required for the environment to exist as it does, fostering the musical and social developments uncovered in this study.

\section{Community of Practice}

Various literatures (Lave and Wenger, 1991; Richards, 2007; Smith, 2005;

Thurber et al., 2007) describe community of practice as a place where a group of people whom share a similar interest come together, forming a community where they engage in developing a set of relationships over time, while collectively learning and sharing information. In this setting members develop a joint set of memories, commitments, and information; a situation that binds them together. Several campers share their perceptions of this community stating:

Here you can play something over and over and no one cares if it doesn't sound very good because everyone is doing the same thing and I like that everyone else is the same interest, everyone is a band nerd. (Camper Interview, italics added)

An entire community of musicians is helpful because there is always something you can learn from someone. (Camper Survey) 
The second statement emerged early in that camper's interview. At the end of the interview when asked if there was anything else about camp he would like to share, he concluded with "I just like it, a community of musicians in the woods" (Camper Interview). It seems that this notion of being in the woods with peers who share a similar interest weighs heavily on why he chooses to return to camp several summers in a row, making a lasting imprint on his view of the camp experience. The combination of forming a community within an environment that completely contrasts that of home creates the joint set of memories that Lave and Wenger talk about.

Lastly, it is also significant to address the fact that a community of practice is also formed through tension. The notions of skill and drive were apparent throughout the study, but presented themselves in two facets. On one hand campers are required to audition upon entrance to camp, serving the function of providing faculty members information on student playing abilities. Based on this information, campers are placed in the "appropriate" upper or lower division of an ensemble, specifically the ensemble the camper had chosen for their schedule. On the other hand, I noticed through observations, many older campers working hard towards this audition with hopes of being with their friends and/or desire to play more advanced music so as to push their abilities. Several younger campers in the pre-high school age range also displayed similar feelings. Some strived for the upper ensembles because they wanted to play with the older campers and believed this experience would stretch their abilities through the challenges of playing harder music with older more advanced musicians. One twelve year trumpet player expressed this feeling describing: 
At home I wouldn't get to play with people that are a lot better than me because everything is segregated into their levels. Here [at camp] there are only two jazz bands. If you get into the upper one and make fourth trumpet you are playing with people who are really really good. (Camper Interview)

This camper mentions "segregation of levels”. While the audition process creates a degree of segregation, the small size of the camp creates a situation where younger players may become members of advanced ensemble sections, interacting socially and musically with their older, more advanced peers. It is important to note that the same opportunity is available to the most advance campers as well, playing in ensembles where they sit side by side the professional musicians of the faculty and staff. The motivation to “compete” for ensemble spots in not so much about winning, but rather, it is rooted in socialization goals and musical aspirations, to receive opportunities where they can tackle new challenges; encounters many of these campers do not experience at school.

The Camp Bubble - A Community Culture. One faculty member spoke of the community feel at camp aptly describing it as the "camp bubble”, explaining: "It's this little universe, this galaxy, our own little contained world” (Faculty Interview). Within this bubble-one can easily observe a certain culture, a way of thinking, a way of interacting, and a way of living that is fostered by the camp directors and ultimately all of the faculty and staff (paraphrased from faculty interview). A faculty member explains this culture thusly:

[The directors] try to and do create a community where we respect people, regardless of how long they've studied music, regardless of what language they speak, regardless of how many years they have been at camp, regardless if they have medical, physical , and social problems, very open minded to all forms of music and all forms of people. (Faculty Interview) 
It seems that a culture of respect, problem solving, and working out issues quickly and efficiently is a necessary component to camp. According to one faculty member:

It is amazing what sets Encore Coda apart; that this kid was having trouble in his cabin, he [also] couldn't really play his trumpet, and within 48 hours of the onset of camp I had met with his counselor, the administration, and two other faculty members. Camp is there for the kids and no other reason, everything is about the kids. (Faculty Interview)

This data show that camp is a student-centered environment, serving the complete needs of the students.

Joseph (2011) asserts that "considering curriculum as culture is a way to attain a holistic understanding of education” (p. 23). The directors at Encore Coda embrace and promote a culture, so much that it deeply influences the curriculum at camp, both the explicit and the hidden. Joseph (2011) furthers that "although individuals will not have identical understanding, the existence of a culture suggests that there are shared systems of meaning” (p. 24), the core of which makes a community of practice. The mix of social and musical interactions at camp creates various curricula outcomes, all stemming from a common culture that is fostered and embraced by the entire community. One faculty member felt that this close knit community environment can also be problematic. The challenge lies in the fact that since everyone is forced to see each other in close proximity, every day and at various times throughout the day, there is a restriction on how intense or how demanding a teacher can be with the students (Faculty Interview, paraphrase). This implies that developmental outcomes are limited due to the culture created at camp, which is an important consideration for future research.

The same faculty member as above contrasted the impact of the community culture at camp to school, stating that unlike traditional school, a teacher at overnight 
camp cannot simply go home at the end of a work day, after a "rough rehearsal” (Faculty Interview, paraphrase). At camp you see everyone after class around camp, in passing on foot, and into the evening when there are camper concerts and various events. This situation forces faculty and campers, faculty and faculty (including counselors), and campers and campers, to work out differences quickly or bite their tongues and keep their true thoughts and emotions inside in order to prevent a problem. Because camp is a small community of constant interactions among the same people, issues or problems that otherwise might be small or avoidable outside the “camp bubble” are commonly transparent and fast acting in camp. This ranges from typical teen drama involving co-ed relationships, rehearsal differences (i.e. clashing of opinions in musical contexts), and homesickness. According to one of the camp directors:

The approach of mediation and dealing with problems is not unique to camp, but what is unique is that at camp all the issues become apparent to everyone within the vicinity very quickly and these matters cannot be hidden or suppressed for long, there is no choice but to deal with them and make things work. (Camp Director Interview)

This statement exemplifies a culture of camp that is a direct result of the camp context. The relation between community, culture, and social relationships formed within the environment create the unique situation and related impacts that occur at this particular overnight summer music camp. The goal presented here is fast problem solving. This particular aim may not be the case elsewhere and is perhaps not a natural experience in all camps, but rather, a result of the particular culture promoted in this particular environment. Further studies would be necessary in order to provide greater insight on this matter. 
One participant who was part of the camper in transition program shared a different angle to the impact of the culture created by the camp’s size stating, “The camp is small enough that the community feels like a family” (Camper Interview). Several other campers expressed a similar view, calling the camp a "home away from home" (Camper Surveys). Consequently, evidence for manifestation of sense of belonging at camp was supported. Within a community of practice every member faces similar situations and challenges, which in turn cultivates the need to support each other and adapt. Barstead (2012) asserts that "humans are social creatures by nature, driven by a need to belong” (para1) and "taking into consideration this need for social connection, it is possible that camps are more essential to the lives of children than ever in our modern culture” (para 2).

\section{Group Size and Development}

Data suggests that the smaller size of Encore Coda allows for the formation of more complex relationships and a greater sense of self -worth or importance for the campers. The notion of group size, or classroom size as referred to in schools, is a relevant issue to education that continues to be researched and experimented with in school settings. The impact of teacher to student ratios youth development within schools has proven to be an ongoing challenge in the field of education. According to Fan (2012) smaller class size leads to more instruction, less discipline, greater academic achievement. The smaller class size also leads to closer relationships and rapport among students as well as students with teachers. Fan (2012) states that in this situation it is easier to identity students’ problem and address them promptly. One participant at Camp Encore Coda describes practices within this community as they relate to student attention: 
They [counselors] have bunk reports every week on every camper, how is this camper interacting with other campers, how are they handling being away from home, how are they dealing with the environment, how do they seem to be musically, are things okay socially but not musically, and they have meetings and discuss how to handle it. I've seen them devise strategies on how to handle it, and divide it up among staff. It all seems they keep their eyes on everything. They seem to always know what is going on. (Faculty Interview)

Overnight summer music camp exists as one alternative learning environment that has overcome the class size obstacle, creating a social and academic platform where participants hold a deeply rooted connection to the other participants, the learning experiences, and the overall community; so much that campers view camp as a home away from home.

Lastly, it is worth commenting on the size of the camp population and the relationships formed within concerns the nature of staff and camper relationships. Just as school, Encore Coda has a clear hierarchy of authority built into the system, one where students respect the "authority" of their counselors, teachers, and administration. The hierarchy also resembles schools on a teacher level, with CIT's reporting to counselors, counselors reporting to head counselors, counselor personnel collaborating with music faculty in order to meet student needs, and ultimately all camp staff reporting to and adhering to the final decisions of the directors. What make the relationships developed at Camp Encore Coda unique is an informality and a democratic understanding of learning, described by one of the camp directors:

Everyone is referred to by their first name. We purposely set aside a lot of the titles that come from the outside world and try and have more natural relationships with people that aren't based on their title. Sometimes you have to invoke your status for certain moments but usually we try to have it be we are all here together, we are all learning together. The informality of it is very important because it fosters lots of information sharing. (Director Interview) 
One camper expresses his perception of this informality asserting "Probably it makes a big difference that they're not Mr. and Mrs. whatever. They are fellow musicians rather than always teacher and student, although it is that too” (Camper Interview). This perception was corroborated by several other campers throughout the data. Viewed in context, it appears that the common understanding that everyone in the community is a learner facilitates relationships which go beyond the traditional teacher-student model, but one where campers can view their teachers as mentors and friends with whom they share a common interest. As the camp director stated before, this close proximity of relationships permits greater levels of information exchange than what would occur in a more formal setting where more rigid barriers are implemented. The nature of this democratic learning culture at Encore Coda is delineated by Allsup and Benedict (2008) who characterize it as "a truly active encounter, one in which there is concern and care between parties, [and] often finds teacher and learner in a horizontal space...away from podiums and seating charts (p. 166).

While Encore Coda certainly has the formal teacher led ensemble rehearsals many students are familiar with, the time "away from the podium and seating charts” represents a time of continued learning. For example:

Staff members who are teaching and giving class, also at lunch one day might mention something to a camper or give them a link they can go to when camp is over online, a performer, or something they didn’t know about. (Director Interview)

Teacher directed learning at Encore Coda, in many cases, contrasts traditional school classrooms through the implementation of a formal, yet unwritten curriculum. While instruction at camp is highly specialized and structured through formal classes, similar to 
what one would see in schools, the content is not pre-determined by administration. According to one faculty member “we don’t really have a curriculum here, teachers can teach whatever they want in classes such as musicianship or piano workshop” (Faculty Interview). The philosophy of the directors is that "the administration trusts the people they hire to impart the knowledge they have been hired to teach” (Faculty Interview). Data suggest overall positive musical learning outcomes from the formal instruction provided at camp, however, a deeper investigation into full teacher empowerment over curriculum would be necessary to identify the more specific outcomes, problems, and challenges of this model.

In sum, the data point to the manifestation of continuous learning as a result of the community created. This camp environ can be qualified as a space where education does not begin and end in the classroom and does not dictate learning the potential outcomes. The formal lessons learned in class, while often rich in content and highly focused, represent just the tip of the iceberg, as student learning extends beyond the walls and into their lives. 


\section{CONCLUSIONS}

The present study explored the niche specialty environment of the overnight music camp. Camp Encore Coda was chosen for this case study; its characteristics are congruent with those of traditional, North American overnight camps, and it is also distinguished by its focus on musical development. The music camp environment derives its uniqueness from many factors such as the natural setting, the staff, the clearly defined daily camper schedule, the interaction between youth living together, the interaction of youth with staff, and the overall structure and philosophy of learning that the camp promotes. At camp, the interactions between youth and staff, all of whom have a common interest in music, can be defined by the concept of a community of practice. According to Richards (2007), communities of practice "are social units that have a common purpose," where "members interact regularly, share common beliefs and vocabulary, and learn from one another as they engage in mutual activities” (p. 40). This theoretical framework guides the study, an investigation into the possible impact a summer music camp has on the attending youth and how such impact is perceived by them. The study is framed by the underlying concepts of multidimensional growth, immersion, communal living, structure in a holistic environment, and socialization, all within the context of a 'community of practice.'

The study was exploratory in nature and designed to provide a better understanding of the music camp environment, hoping to uncover what makes it unique, how learning occurs, and how the youth perceive the experience of attending a session. Emphasizing the musical context, this study interrogated the assertions of Bhattacharyya, Nathaniel, and Mead (2011), who argue that unique camp environments are those where 
youth are given the opportunity to develop in ways that they do not in the traditional classroom. The present investigation is particularly significant for music educators who strive to understand the role of context and pedagogical orientation upon musical growth, given the dearth of research on the educational and social effects of music camps.

This qualitative case study consisted of a process where I gathered data using multiple entry points into the environment, using triangulation to establish the study's validity (Merriam, 1988). The instruments used included surveys, interviews, observations, and one focus group. Participants included youth in the upper camp division, though I observed all campers. I collected data onsite at Camp Encore Coda, located in Sweden, Maine over the final week of the camp's last session of the 2013 summer, August 4-10.

I analyzed the data using axial coding; organizing the emerging themes into categories, thereby presenting a clear picture of how each element is interconnected in the larger context (Strauss and Corbin, 1998). While the data paint a mostly positive picture of what takes place at camp Encore Coda, it is important to note the high level of selfselection that inheres in such studies tends to skew some of the data, which therefore must be taken with caution. Moreover, data may be shaped by any number of external factors including but not limited to the socioeconomic status (SES) and prior life experiences of participants, as well as the information the researcher chooses not to include in the findings and analysis. 


\section{Addressing Research Questions}

The following research questions framed this research study:

Research Question 1. In what ways, if any, does overnight music camp facilitate musical and personal growth?

The key element to emerge out of this question is the link between the formation of a community of practice and facilitation of musical and personal growth. Within this community, students had shown themselves driven by different motivations: some seek to improve their musicianship, some hope for new experiences, some anticipate the exhilarating social interactions that might result from being away from home, in the woods, with peers who also love making music. Regardless of the campers’ intent, they are all connected by music-making in a communal living context. Music is a common thread attracting the youth to a learning environment that reaps multidimensional growth outcomes. A key notion underlying participation in this context is that the youth want to be there. The initial motivation to attend camp is further propelled by the marked growth experienced in a new environment with musical progress and the formation of social relationships as the underlying stimuli. Desire to attend camp supports the multi-layered levels of growth campers experience during their three weeks onsite and is connected to the major themes uncovered in this study. Immersion-enculturation, communal living, structure, isolation, and learner agency are among the important themes that emerge from this study.

Benedict (2009) eloquently presents a description that illuminates the significance of the communal learning environment established at camp, stating, “These aren’t just communities of young vibrant musicians of all kinds; rather, these are vibrant musicians 
who engage critically with the world around them and who realize potentiality beyond musical engagements” (p. 164). The formation of multifaceted social relationships within a continuously busy daily routine, form the basis for both the social and musical skills development experienced by campers, consequently providing a place where youth can stretch themselves and discover the abilities they may not have recognized prior to the camp experience.

Through the established structure and self-reliance formed at camp augmented by complex interactions, youth develop a sense of belonging. Growth is both sociological and skill based, linking back to a holistic education allowing students to experience a more diverse and complex kind of learning. The learning outcomes can be traced to both the intensive daily instruction and informal learning, often peer driven or unplanned stemming from interactions that occur throughout the day in and out of classes. The intersection of “academic learning” with socialization follows Schmidt’s (2012) assertion that "musical education must be interwoven with other concerns and other more encompassing constructs if it is to build robust, meaningful, and complex learning outcomes” (p. 52).

Research Question 2. In what ways, if any, does the learning experience at overnight music camp differ from that of the traditional classroom?

The growth participants experience during their time at camp reinforces preexisting knowledge, building on familiarity, while also creating a space to try new things and make decisions they normally do not have the opportunity to make during the rest of the year. There are several distinct ways the learning experiences at Camp Encore Coda parallel and contrast those of the traditional classroom. The perceptions and overall 
feelings campers share concerning the various facets of camp illustrate why they attend, what makes camp unique, and how they view camp compared to their traditional classrooms at home. Camp exists as an extension of their learning in school, presenting opportunities to develop through the support of a community of practice. While schools have and will retain their own, often rigid, structures, the data from this study suggests that finding ways to replicate the sense of connection and ownership that many campers yearn for is worth pursuing. Within Encore Coda, campers exhibit ownership over their musical learning as well as the community, taking pride in belonging to a place they feel they can fit in and thrive.

Unlike school, however, camp does not lack musical options for the youth. These options exist on a social level, encompassing daily choice making, as well at a musical level, concerning ensembles, repertoire, and choices that occur within these varied situations. The wide array of options at camp comes as a unique aspect to this environment, one that perhaps cannot occur in another context where multiple academic disciplines are also studied.

This leads us to the notion of time, an ever so present challenge in schools. At camp, continuous and intense learning is collapsed into three weeks. The musical education is interwoven with social concerns and consequently fosters information sharing from the moment campers wake up to when they finally go to sleep at night. At school student learning typically ends when the bell rings. The absence of "learning time" limitation in the camp context affords students ample amount of time to perform teacher and self-assigned tasks in a considerably shorter time frame than they would in school settings. 
Lastly, at camp students push themselves in ways they may not at home. This particular kind of motivation is evident in the analysis and seems to emanate from the community support. The external motivation of community builds upon the every student's natural enjoyment of music. Compared to school camp offers new experiences in the context of a low pressure, noncompetitive environment, yet, yields more complex, highly focused engagements and relationships than normally occurs in the school classroom, although understanding the depth of engagement in all schools cannot be established without full analysis of specific schools. Finally, comparative findings in this study are solely based on the experiences and reports by Camp Encore participants regarding their schools at home.

Research Question 3. In what ways, if any, do participating youth perceive and articulate these possible differences?

The structure and pedagogical orientation of school classrooms exhibit distinct contrasts to those of camp. It is the intersection of the familiar and unfamiliar that defines this particular learning environment. The youth realize that through the intersection of different and varied pedagogical approaches in a more holistically grounded context, they have opportunities to grow in ways they do not during the other months of the year. They seem quite aware of this distinction.

Campers felt the support of living and working in a community of practice and expressed heightened motivation from their experience in an environment where everyone practiced music. Within this context the youth look up to their teachers, feeding off of their shared energy and passion for music. Campers found that the relationships formed resulting from environmental and camp cultural influences were significant. The 
lack of formality regarding everyone, including faculty referred to on a first name basis furthered the connection campers felt to the community. As a result, camper-staff relationships fostered multidimensional learning, which was well perceived by the campers, who felt comfortable seeking advice from their mentors inside and outside of class. All these constituted environmental elements that defined the perceived differences between school and camp, as established by the campers themselves.

The youth further realized the significance of functioning as their own learning agent, embracing the opportunities at camp to make decisions they normally cannot make at home or in school. Campers felt empowered by these opportunities, realizing that they were capable of learning on their own, and accomplishing tasks they may have not attempted or thought they could conquer in the past. The sense of confidence was demonstrated in their performances and constant engagement with peers and staff around camp.

Lastly, the notion of ownership weighed heavily upon the campers' perceptions of the overnight experience and the learning opportunities within. As active members in an open and perhaps democratic learning community, the youth perceived both their work and the environment as something personal. From the performances and compositions to the daily responsibilities with living communally in a cabin, the youth at Camp Encore Coda take pride in their efforts, especially when they have overcome challenging obstacles.

\section{Unanticipated Themes}

While this study explored the majority of significant themes pertaining to overnight music camps in some detail, particularly of a positive nature, issues I did not 
anticipate and nor have time to investigate still remain. Some are negative. These include time constraints, the notion of "forced" practice, and the challenges of an unwritten curriculum that is consequently unevenly enforced.

\section{Implications for Future Research}

The significance of this research lies not only in uncovering and addressing the present questions, but also the opening of doorways for future investigative endeavors. Its detailed exploration of one camp has paved the way for future research regarding camps environs as well as pedagogical or curricular issues found within them.

First, expanding on the data collected in this study, a future study could seek parental perceptions of campers, exploring what they feel the impact of camp is upon the growth of their children. As the "payers" who send their children away for the summer, and in many cases multiple summers in a row, it would be interesting to inquire why parents feel camp is "worthwhile.”

Second, camp is not only a place for development among youth, but also a breeding and training ground for young as well as experienced teachers. According to Richards (2007), training teachers to be prepared for working with diverse populations is an existing challenge in our educational system. The significance of the time teachers spend teaching music in the summer months may not only impact the youth at the camps, but also the quality of our entire music education system for it facilitates teachers’ consistent sharpening of their skills in a variety of educational settings. Additional studies, such as the investigation of the staff experience would be a meaningful extension of this initial survey. 
Though this study examined only one overnight music camp, several other North American camps exhibit many of the same characteristic as Encore Coda while maintaining their own unique traits. A comparative study of other camps would expand the current findings, broadening the way we see how camps function and exist as a significant part of music education.

Lastly, more specific issues concerning music learning emerged in the data and stand as worthy constructs for additional research. One of these is the issue of ordering social interaction with or through music. Further exploration might offer insight into the social as well as subjective lives of youth interested in music. Furthermore, this kind of purposeful social ordering leads us to consider the notion of agency and the multifaceted ways in which it can be constructed by youth.

Representing just a few potential pathways for future investigation, there numerous lenses form which a researcher can pursue related work in the future. The exploration of Camp Encore Coda uncovered environmental and relational complexities that transcend solely one context, but rather, expose possibilities for future research and applications of findings to educational contexts of all kinds.

\section{Final Remarks}

The data and analysis presented here might compel music educators to consider that schools should design music curriculum not only for passionate musicians, but as a way of sparking learning engagement for multitudes of youth, incorporating interdisciplinary connections in the classroom so that students who pursue music after high school and those who don't can reap the long-term learning benefits in class. Rather 
than students omitting music from their schedule because they are interested in foreign language or history and do not have "the time to waste," these students can embrace the enjoyment aspect of music while simultaneously growing in their other subjects they plan to ultimately pursue professionally.

The findings in this study substantiate the great importance environment and pedagogical approach has on learning. Environment creates a platform for learning that fosters a variety of learning opportunities through the numerous engagements between student and teacher, student and peers, and student and self. Music camp supports the notion that there is no one correct way for people to learn, but rather, a wide range of possibilities based on environmental factors and the unique abilities and interests of each individual. Encore Coda facilitates these possibilities through the creation of a community of practice that operates in the complex learning environment known as overnight summer music camp. Within this environment a diversity of teaching approaches are presented to the students through the implementation of an unwritten curriculum. Each teachers professional experience and teaching style contributes to the formation of a place where student centered education is at the core.

Camp Encore Coda has created an experience that caters to a diversity of youth from different cultures, socioeconomic status, and musical abilities, some who go on to pursue music, and many who forge ahead into other fields of study such as math, science, writing, and technology. Much deeper than its impact on the improvement of musical and social skills, the camp is a place that nurtures and catapults tomorrow's thinkers out into the world. Providing much more than simply a new learning opportunity, overnight music camp creates a second home for many of its participants, a place where learning and 
socialization are conjoined, where individuals can embrace their own passions and

dreams, sharing them with others without judgment, enabling the youth to grow into their own shoes in a way that works for them. 


\section{REFERENCES}

Adams, M. (2009).Finding my voice as a qualitative researcher: An autoethnographyabout learning to trust myself as a kid watcher.Journal of Reading Education, 34(3), 8.

Allsup, R. E., \& Benedict, C. (2008). The problems of band: An inquiry into the future of instrumental music education. Philosophy of Music Education Review,16(2), 156-173.

Babbie, E. R. (1990). Survey research methods (Second ed.). Belmont, Calif.: Wadsworth.

Barstead, M. (2012, Jul. - Aug.). The human need to belong: An evolution in how we think about camps. Camping Magazine. Retrieved April 24, 2014, from http://www.acacamps.org/campmag/1207/human-need-belong

Baumeister, R., \& Leary, M. (1995). The need to belong: Desire for interpersonal attachments as a fundamental human motivation. Psychological Bulletin, 117(3), 497529.

Belin, L. R. (2009). Exploring the experiences of three teachers at a summer youth music camp: "As positive for the faculty as it is for the kids". (Unpublished masters dissertation). University of Maryland, College Park, Md.

Benedict, C. (2009). Designing music curriculum. In H. Abeles \& L. Custodero (Eds.) Music pedagogy: Frameworks for practice. New York, NY: Oxford University Press.

Bhattacharya, S., Nathaniel, R., \& Mead, T. (2011).The influence of science summer camp on African-American high school students' career choices.School Science and Mathematics, 111(7).

Bieleschki, D. M., Younger, T., Henderson, K., Ewing, D., \& Casey, M. (2002, Jan.Feb.). Happy but sad: Outcomes at Morry's Camp. Camping Magazine, Retrieved April 24, 2014, from http://www.acacamps.org/members/knowledge/participant/cm/021happy

Boud, D., Cohen, R., \& Sampson, J. (1999). Peer learning and assessment. Assessment \& Evaluation in Higher Education, 24(4), 414-426.

Brandt, T. A., (1988). Summer music camps: A historical perspective. The Bulletin of Historical Research in Music Education, 9(2), 119-130.

Chichuki, P. (2007).What is happening to me.Montessori Life: A Publication of the American Montessori Society, 19(4) 20-21. 
Cohen, L., Manion, L., \& Morrison, K. (2011). Research methods in education (Second ed.). New York: Routledge.

Creswell, J. (2003). Research design: Qualitative, quantitative, and mixed methods approaches (2nd ed.). Thousand Oaks, CA: Sage Publications.

Creswell, J. (1998). Qualitative inquiry and research design: Choosing among five traditions. ThousandOaks, CA: Sage.

Cueto S., Guerrero G., Sugimaru C., \& Zevallos A. M. (2010). Sense of belonging and transition to high schools in Peru.International Journal of Educational Development. 30, 277-287.

DeNora, T. (2000).Music in everyday life.Cambridge University Press.

Denzin, N. K., \& Lincoln Y. S. (2003).Collecting and interpreting qualitative materials (2nd ed.) Thousand Oaks, CA: Sage.

Denzin, N. K., \& Lincoln, Y. S. (1994). Handbook of qualitative research.Thousand Oaks, CA: Sage Publications, Inc.

Dewey, J. (1987). John Dewey's theory of art, experience, and nature : the horizons of feeling. Albany : State University of New York Press.

Dewey, J. (1916). Democracy and education (1966 ed.). New York: Free Press.

Dewey, J. (1938). Logic, the theory of inquiry. New York : H. Holt and Company.

Eisner, E. W. (2002). The kinds of schools we need. The arts and the creation of mind (pp. 576-583). New Haven: Yale University Press.

Diaz, F. M., \&Silveira, J. (2013).Dimensions of flow in academic and social activities among summer music camp participants. International Journal of Music Education, 31(3), 310-320.

Fan, F. A. (2012). Class size: Effects on students' academic achievements and some remedial measures. Research in Education , 87, 95-98.

Farris, R. (n.d.). Why are kids coming to camp and why are their parents sending them there?. Washington Family Magazine. Retrieved November 12, 2012, from http://www.washingtonfamily.com/page/Why-Are-Kids-Coming-to-Camp-and-WhyAre-Their-Parents-Sending-Them-There

Gerring, J. (2004, May). What is a case study and what is it good for?.American Political Science Review,98(2). 
Gillard, A., Roark, M. F., Nyaga, L. R. K., \&Bialeschki, D. M. (2011).Measuring

mindfulness in summer camp staff.Journal of Experiential Education, 34(1), 87-95.

Goodenow, C. (1993). Classroom belonging among early adolescent students:

Relationships to motivation and achievement. The Journal of Early Adolescence, 13(21).

Green, L. (2008). Music, informal learning and the school: a new classroom pedagogy. Burlington, VT: Ashgate Publishing Company.

Greene, M. (1978).Steamboats and critiques. Landscapes of learning (pp. 111-125). New York: Teachers College Press.

Hall, J. (2008). Cross-sectional survey design. In P. Lavrakas (Ed.), Encyclopedia of survey research methods. (pp. 173-174). Thousand Oaks, CA: SAGE Publications, Inc.

Hall, J. L. P. (2000). The international music camp: Model band camp from an historical perspective (Doctoral dissertation). Retrieved from ProQuest Dissertations http://hdl.handle.net/11244/5963

Hampton, C. (2008). A case study of a summer music camp and the impact of participation on perceptions of the college of music and the hosting university (Doctoral dissertation). ProQuest Dissertations and Theses http://diginole.lib.fsu.edu/etd/4299

Hickey, M., \& Webster, P. (2001, July).Creative thinking in music.Music Educators Journal, 88, 17-18.

Instrumental Programs.(n.d.)Berklee College of Music. Retrieved February 14, 2014 from http://www.berklee.edu/instrumental-programs

Jansen, H. (2010). The logic of qualitative survey research and its position in the field of social research methods. Forum Qualitative Sozialforschung / Forum: Qualitative Social Research, 11(2).

Juvonen, J. (2006). Sense of belonging, social bonds, and school functioning. In P. Alexander, \& P. Winne (Eds.), Handbook of Educational Psychology.(2nd ed., pp. 655674). New York: Macmillan.

Kelly, S. N., \&Juchniewicz, J. (2009).An investigation of social and musical objectives and experiences sought by instrumental students attending a summer music camp. In S. Cooper (Dir.), The Proceedings of the Eleventh Biennial Desert Skies Symposium (6576).Tucson, AZ: University of Arizona School of Music. 
Kidder, L. (1981).Qualitative research and quasi-experimental frameworks. In M. Brewer et al. (Eds.), Scientific inquiry and the social sciences (pp. 227-256). San Francisco: Jossey-Bass.

Kirschman, K. J. B., Roberts, M. C., Shadlow, J. O., \&Pelley, T. J. (2010).An evaluation of hope following a summer camp for inner-city youth. Child and Youth Care Forum, 39(6), 385-396.

Krueger, R.A., \& Casey, M.A. (2009).Focus groups: A practical guide for applied research (4th ed.).Thousand Oaks, CA: Sage Publications.

Larson R. (2000). Toward a psychology of positive youth development.American Psychology 55, 170-183

Lave, J. and Wenger, E. (1991) Situated learning. Legitimate peripheral participation.Cambridge: University of Cambridge Press.

Managan, M. G. (1989). The impact of the traditional classroom environment vs. the informal classroom environment on creative thinking abilities. Nacogdoches, Texas: Stephen F. Austin State University.

Markham, L. (n.d.). Structure: Why kids need routines. Aha! Parenting. Retrieved April 24, 2014, from http://www.ahaparenting.com/parenting-tools/family-life/structureroutines

Merriam, S.B. (1998).Qualitative research and case study application in education. San Francisco: Jossey Bass Publishers.

Merriam, S.B. (1988).Case study research in education: A qualitative approach. San Francisco: Jossey-Bass Publishers.

Miller, R. (1992). What are schools for? Holistic education in American culture.Brandon, Vermont: Holistic Education Press.

Modell, H. I., DeNiero, G., \&Rose, L. (2009).In pursuit of a holistic learning environment: the impact of music in the medical physiology classroom.Advances in Physiology Education 33, 37-45.

Morgan, D. L.(1996).Focus groups. Annual Review of Sociology, 22, 129.

National Camp Association.(1987). Trends in summer camp.Retrieved from http://www.summercamp.org/media/article3.html, Nov. 12, 2012. 
Ozier, L. (2009, Nov.-Dec.).Camps and classrooms.Camping Magazine. Retrieved February 1, 2014 from http://www.acacamps.org/campmag/issues/0911/camps-andclassrooms

Richards, J. (2007). A community of practice summer literacy camp: One way to connect preserviceteachers, graduate education majors, and elementary children. Journal of Reading Education, 32(2).

Rubin, K. H., Bukowski, W., \& Parker, J. G. (1998). Peer interactions,relationships, and groups. In W. Damon \&N.Eisenberg (Eds.), Handbook of child psychology: Vol. 3. Social,emotional, and personality development (5th ed., pp. 619-700). New York: Wiley.

Schmidt, P. (2012). Music, Policy and Place Centered Education: Finding a Space for Adaptability. National Society for the Study of Education. Teachers College Press.

Seidman, I. E. (1991). Interviewing as qualitative research. New York: Teachers College Press

Sherwood, A. (2005). Never too old for camp. Retrieved November 12, 2012 from http://www.summercamp.org/media/article17.html

Smith, M. (2005).Communities of practice.The encyclopedia of informal education.Retrieved February 10, 2014from www.infed.org/biblio/communities_of_practice.htm

Stake, R. E. (2000).Case studies. In N.K. Denzin\& Y.S. Lincoln (Eds.), Handbook of qualitative research (pp. 435-454). Thousand Oaks, CA: Sage Publishing, Inc.

Strauss, A., \& Corbin, J. (1998). Basics of qualitative research: Techniques and procedures for developing grounded theory. Thousand Oaks, CA: Sage.

Thurber, C. A., Scanlin, M. M., Scheuler, L., \& Henderson, K. A. (2007). Youth development outcomes of the camp experience: Evidence for multidimensional growth. Journal of Youth and Adolescence, 36(3), 241-254.

Timeline.(n.d.). American Camp Association. Retrieved November 30, 2012, from http://www.acacamp.org/anniversary/timeline/

Tirri, K. (2011). Holistic school pedagogy and values: Finnish teachers' and students' perspectives. International Journal of Educational Research, 50(3), 159-165.

Webster, P. (1990). Creativity as creative thinking.Music Educators Journal, 76(9). 
Westervelt, V. D., Johnson, D. C., Westervelt, M. D., \&Murrill, S. (1998). Changes in self-concept and academic skills during a multimodal summer camp program. Annals of Dyslexia, 48, 191-212.

Wiggins, J. (2011). When the music is theirs: Scaffolding young songwriters. In M. Barrett (Ed.), Aculturalpsychology for music education (pp. 83-113). London: Oxford University Press.

Yin, R. K. (2003).Case study research: Design and methods (3rd ed.). Thousand Oaks,CA: Sage.

Yin, R.K. (1984).Case study research: Design and methods. Newbury Park, CA: Sage. 


\section{APPENDICES}




\section{APPENDIX A}

\section{Consent Forms}

\section{FLORIDA
INTERNATIONAL
UNIVERSITY}

\section{CHILD ASSENT TO PARTICIPATE IN A RESEARCH STUDY}

\section{A Case Study: Overnight Summer Music Camp and the Impact on its Youth}

\section{WHY ARE YOU DOING THIS STUDY?}

We would like for you to be in a research study we are doing. A research study is a way to learn information about something. We would like to find out more about the music camp environment and gain a better understanding of how it might uniquely impact the musical and social development of its attending youth.

\section{HOW MANY OTHERS WILL BE IN THIS STUDY?}

If you agree to participate in this study, you will be one of approximately 150 children in this research study.

\section{HOW LONG WILL THE STUDY LAST?}

Your participation will require your attendance for one full session at Camp Encore Coda. The study will not interrupt your daily activities at camp. Your participation will include completing a survey at the beginning of camp and at the end of camp, each taking about 20-30 minutes. If you are asked to do an interview or a group discussion this may take 20 -30 minutes as well. You may choose to do the survey but decline the other parts of the study for any reason as all parts are voluntary.

\section{WHAT WILL HAPPEN IN THIS STUDY?}

If you participate in this study, we will ask you to do the following things:

-Take two surveys

-Be observed during your camp activities

-Possibly answer some interview questions (may only apply to select campers) 
-Participate in a group discussion (may only apply to select campers

\section{CAN ANYTHING BAD HAPPEN TO ME?}

There are no known risks to you associated with this study.

\section{CAN ANYTHING GOOD HAPPEN TO ME?}

The following benefits may be associated with your participation in this study:

-Improved music education in your schools

-Greater opportunities for you and other youth to attend camp in the future

-Promoting of Summer Music Camps

\section{DO I HAVE OTHER CHOICES?}

There are no known alternatives available to you other than not taking part in this study.

\section{WILL ANYONE KNOW I AM IN THE STUDY?}

The records of this study will be kept private and will be protected by the researchers. Your name will not be used in any of the data collected and all information from surveys, observations, discussions, and interviews will be kept completely confidential.

\section{WILL I BE GIVEN ANYTHING FOR PARTICIPATING?}

There is no payment for this study. You will not need to pay for anything to participate in this study.

\section{WHAT IF I DO NOT WANT TO DO THIS?}

You do not have to be in this study if you don't want to and you can quit the study at any time. If you don't like a question, you don't have to answer it and, if you ask, your answers will not be used in the study. No one will get mad at you if you decide you don't want to participate.

\section{WHO CAN I TALK TO ABOUT THE STUDY?}

If you have any questions about the research study you may contact Ari Nemser at Miami, Fl, 609-462-7342 ANEMS001@FIU.EDU. If you would like to talk with someone about your rights of being a participant in this research study, you may contact the FIU Office of Research Integrity by phone at 305-348-2494 or by email at ori@fiu.edu.

\section{PARTICIPANT AGREEMENT}

This research study has been explained to me and I agree to be in this study. 
Signature of Child Participant

Printed Name of Child Participant

Signature of Person Obtaining Consent
Date

Date 


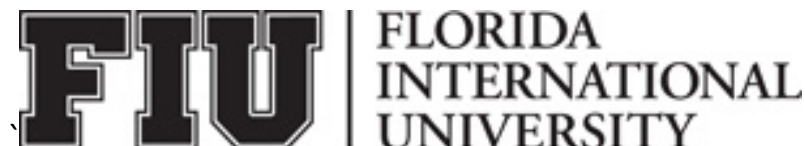

\section{PARENTAL CONSENT TO PARTICIPATE IN A RESEARCH STUDY}

A Case Study: Overnight Summer Music Camp and the Impact on its Youth

\section{PURPOSE OF THE STUDY}

You are being asked to give your permission for your child to be in a research study. The purpose of this study is to explore the music camp environment and gain a better understanding of how it might uniquely impact the musical and social development of its attending youth.

\section{NUMBER OF STUDY PARTICIPANTS}

If you agree to allow your child to participate in this study, he/she will be one of approximately 150 youth in this research study.

\section{DURATION OF THE STUDY}

Your child's participation will require he/she attend at least one full session at Camp Encore Coda. The study will not interrupt daily activities at camp. Participation will include completing a survey at the beginning of camp and at the end of camp, each taking about 20-30 minutes. If asked to do an interview or a group discussion this may take 20 -30 minutes as well. Campers may choose to do the survey but decline the other parts of the study for any reason as all parts are voluntary.

\section{PROCEDURES}

A significant portion of the information collected for this study will be acquired through unobtrusive observations of your child in his/her various camp activities. However, if your child participates in this study, we might also ask your child to do the following things:

1. Campers will be asked to fill out two brief surveys. These surveys will include questions about prior camp experiences, prior music experience, what instrument they play, their perceptions on what and how they have learned music in the past during camp, and how they have learned music in their school environments.

2. Some selected campers may be asked to participate in short, informal interviews where they will be asked about their various camp experiences. Interviews will never last longer than 30 minutes. Student's may refuse to participate in answering any questions or may completely refuse interview participation if preferred. Interviews will likely be recorded or 
written down for the purpose of accurate data collection. No one other than the researcher and his advisor will have access to recordings. Student anonymity and confidentiality will be assured.

\section{RISKS AND/OR DISCOMFORTS}

There are no known risks associated with this study for your child.

\section{BENEFITS}

Participants will not directly benefit from this study. The following benefits, however, may be associated with your child's participation in this study: Your child will have the opportunity to participate in a seminal study of Camp Encore Coda aiming to uncover the impact of the camp experience on its youth. Data gathered through student participation will potentially inform families, youth, and educators across the United States regarding musical practices in Summer Camps. Implications of this study will range from greater awareness and advocacy for overnight summer music camps, pursuits of further related studies, and overall improvement of music education in the United States.

\section{ALTERNATIVES}

There are no alternatives available to your child other than not taking part in this study. Not participating in this study will have no negative impacts on your child's experience during summer camp.

\section{CONFIDENTIALITY}

The records of this study will be kept private and will be protected to the fullest extent provided by law. Any and all reports that might be published as an outcome of this study will exclude any information that might make it possible to identify your child as a subject. Research records will be stored securely and only the researcher will have access to the records. However, authorized University personnel or other agents who will be bound by the same provisions of confidentiality may review your child's records for audit purposes.

\section{COMPENSATION\& COSTS}

You and your child will not be responsible for any costs to participate in this study. No compensation will be offered to any of the participants, their parents or legal guardians.

\section{RIGHT TO DECLINE OR WITHDRAW}

Your child's participation in this study is voluntary. Your child is free to participate in the study or withdraw his/her consent at any time during the study. Your child's withdrawal or lack of participation will not affect any benefits to which he/she is otherwise entitled. The investigator 
reserves the right to remove your child from the study without your consent at such time that they feel it is in the best interest.

\section{RESEARCHER CONTACT INFORMATION}

If you have any questions about the purpose, procedures, or any other issues relating to this research study you may contact Ari Nemser at Miami, Florida, 609-462-7342, ANEMS001@FIU.EDU or Professor Patrick Schmidt, at pschmi@fiu.edu or 609-532-6316.

\section{IRB CONTACT INFORMATION}

If you would like to talk with someone about your child's rights of being a subject in this research study or about ethical issues with this research study, you may contact the FIU Office of Research Integrity by phone at 305-348-2494 or by email at ori@fiu.edu.

\section{PARTICIPANT AGREEMENT}

I have read the information in this consent form and agree to allow my child to participate in this study. I have had a chance to ask any questions I have about this study, and they have been answered for me. I understand that I am entitled to a copy of this form after it has been read and signed.

Signature of Parent/Guardian

Date

Printed Name of Parent/ Guardian

Printed Name of Child Participant

Signature of Person Obtaining Consent

Date 


\section{FIU]}

\section{Staff CONSENT TO PARTICIPATE IN A RESEARCH STUDY}

\section{A Case Study: Overnight Summer Music Camp and the Impact on its Youth}

\section{PURPOSE OF THE STUDY}

You are being asked to give your permission to be in a research study. The purpose of this study is to explore the music camp environment and gain a better understanding of how it might uniquely impact the musical and social development of its attending youth.

\section{NUMBER OF STUDY PARTICIPANTS}

If you agree to participate in this study, you will be one of approximately 200 people in this research study, a culmination of approximately 150 campers and 50 staff.

\section{DURATION OF THE STUDY}

Your participation will require you work at least one full session at Camp Encore Coda. Should you agree to participate you may be asked to be interviewed about camp in a time frame of 2030 minutes that does not conflict with your work duties.

\section{PROCEDURES}

A significant portion of the information collected for this study will be acquired through unobtrusive observations of campers and staff in their various camp activities. However, a select group of staff/faculty may be asked to do the following:

1. Some selected staff/faculty may be asked to participate in short, informal interviews where you will be asked about your various camp experiences. Interviews will never last longer than 30 minutes. You may participate in answering any questions or may completely refuse interview participation if preferred. Interviews will likely be recorded or written down for the purpose of accurate data collection. No one other than the researcher and his advisor will have access to recordings. Participant anonymity and confidentiality will be assured.

\section{RISKS AND/OR DISCOMFORTS}

There are no known risks associated with this study. 


\section{BENEFITS}

Participants will not benefit directly. The following benefits however may be associated with your participation in this study: You will have the opportunity to participate in a seminal study of Camp Encore Coda aiming to uncover the impact of the camp experience on its youth. Data gathered through your participation will potentially inform families, youth, and educators across the United States regarding musical practices in Summer Camps. Implications of this study will range from greater awareness and advocacy for overnight summer music camps, pursuits of further related studies, and overall improvement of music education in the United States.

\section{ALTERNATIVES}

There are no alternatives available to you other than not taking part in this study. Not participating in this study will have no negative impacts on your experience during summer camp.

\section{CONFIDENTIALITY}

The records of this study will be kept private and will be protected to the fullest extent provided by law. Any and all reports that might be published as an outcome of this study will exclude any information that might make it possible to identify you as a subject. Research records will be stored securely and only the researcher will have access to the records. However, authorized University personnel or other agents who will be bound by the same provisions of confidentiality may review records for audit purposes.

\section{COMPENSATION\& COSTS}

You will not be responsible for any costs to participate in this study. No compensation will be offered to any of the participants.

\section{RIGHT TO DECLINE OR WITHDRAW}

Your participation in this study is voluntary. You are free to participate in the study or withdraw consent at any time during the study. Your withdrawal or lack of participation will not affect any benefits to which you are otherwise entitled. The investigator reserves the right to remove you from the study without your consent at such time that they feel it is in the best interest.

\section{RESEARCHER CONTACT INFORMATION}

If you have any questions about the purpose, procedures, or any other issues relating to this research study you may contact Ari Nemser at Miami, Florida, 609-462-7342, ANEMS001@FIU.EDU or Professor Patrick Schmidt, at pschmi@fiu.edu or 609-532-6316.

\section{IRB CONTACT INFORMATION}


If you would like to talk with someone about your rights of being a subject in this research study or about ethical issues with this research study, you may contact the FIU Office of Research Integrity by phone at 305-348-2494 or by email at ori@fiu.edu.

\section{PARTICIPANT AGREEMENT}

I have read the information in this consent form and agree to participate in this study. I have had a chance to ask any questions I have about this study, and they have been answered for me. I understand that I am entitled to a copy of this form after it has been read and signed.

Signature of Participant

Printed Name of Participant
Date

\section{Date}




\section{APPENDIX B}

\section{SURVEYS}

\section{Preliminary Survey for Camp Encore Coda Research Study}

\section{Dear Campers,}

This survey will assist in a first time research study of Camp Encore Coda. The information gained from this survey and from the data collected over the summer will help in discovering details about participants' perceptions about this music camp, furthering the development of music education, and improving musical opportunities for youth in the future. Your views are therefore essential in fully understanding the camp experience from a musical, social and personal standpoint. I hope you will help us in this enterprise!

Please answer each question thoughtfully and carefully with as much detail as necessary. You may choose not to participate in this study or not to answer any particular questions, as this is a voluntary study. The names of all participants will be kept anonymous, as will any of the answers you provide. Your participation is greatly appreciated.

For any further questions please do not hesitate to contact me in person at camp or via phone or email.

Thank You,

$$
\text { Qrip Pamser }
$$

Ari Nemser

6094627342

ANEMS001@FIU.EDU 
Name:

Instrument:

Age:

Session: 1, 2, or both (circle)

First time Camper at Encore Coda? Yes No

If past camper, how many summers have you attended in the past? $1 \quad 2 \quad 3 \quad 4+$

Please answer the following questions with a rating scale by circling the appropriate box.

1 =not at all 2=not much 3=good amount 4=very much so

$5=\mathrm{A}$ lot

How much do you expect to improve on your instrument this summer?

$\begin{array}{lllll}1 & 2 & 3 & 4 & 5\end{array}$

How much do you expect to improve your overall musicianship?

$\begin{array}{lllll}1 & 2 & 3 & 4 & 5\end{array}$

Do you expect to learn anything this summer you normally do not learn during your regular school year?

$\begin{array}{lllll}1 & 2 & 3 & 4 & 5\end{array}$

To what extent do you learn about music from your parents?

$\begin{array}{lllll}1 & 2 & 3 & 4 & 5\end{array}$

To what extent do you learn about music from school teachers?

$\begin{array}{lllll}1 & 2 & 3 & 4 & 5\end{array}$

To what extent do you learn about music from your peers at home/in school?

$\begin{array}{lllll}1 & 2 & 3 & 4 & 5\end{array}$

Do you expect to make any lasting friendships?

$\begin{array}{lllll}1 & 2 & 3 & 4 & 5\end{array}$ 
Do you expect to improve yourself at camp this summer in any non-musical areas?

$\begin{array}{lllll}1 & 2 & 3 & 4 & 5\end{array}$

Please Answer In Complete Thoughtful Sentences:

Why did you choose to attend Encore Coda? How was the selection process made? Please be specific

Please describe your overall musical background i.e. when you first took any formal classes, experience at home, in the community, or at school.

What do you like or dislike about music at your school and why?

What about the overall camp environment appeals to you? What kinds of activities are you looking forward to? Which are your favorites?

Describe an outstanding musical experience you have had at Encore Coda or any other camp. 
What musical activities do you expect to participate in at camp this summer?

What are your greatest musical strengths? If you are a returning camper, has the camp had an impact on those? Explain

What are your greatest musical weaknesses? If you are a returning camper, has the camp had an impact on those? Explain

Do you see any connections between the camp environment and your musical learning? Explain

What are your greatest non-musical personal strengths? 
What are your greatest non-musical weaknesses?

What non-musical activities do you participate in during the regular school year? i.e. sports, clubs, youth organizations

What non-musical activities do you hope or expect to participate in at camp this summer? Circle Any

swimming basketball tennis lawn games

hiking boating ping pong model rocketry

arts and crafts volleyball softball kickball other(please specify)

What areas of personal growth do you hope or expect to improve upon this summer? Please be specific

What concerns, if any, do you have about your camp experience this summer?

What do you expect to gain from camp that you cannot gain or experience during the regular school year musically or socially? Please be specific and detailed 


\section{Camp Encore Coda Closing Survey 2013}

Please answer with complete and thoughtful responses. Use as much space as you need.

1) Tell a brief story that explains what camp was like for you this summer.

2) Name five things you learned at camp that are important to you.

3) What part of camp, negative or positive, sticks out in your mind the most?

4) If you could change one thing about your camp experience what would it be?

5) In what ways, if any, did you learn from your peers this summer? 
6) Engagement is being extremely focused, attentive, interested, and connected with an activity, experience, or conversation. Think back to your summer, when were you the most engaged?

7) Describe when and where this summer you were given the opportunity to make decisions (as opposed to being told or directed what to do by an authority figure). How did this make you feel?

8) In what ways did you grow as a musician at camp?

9) Did being at camp this summer change you in any ways? If so, what ways? 
10) Explain in your own words, how life and music during camp are different from the regular year.

11) What would you say about camp to a future potential camper who has never attended before?

12) Why would you or why would you not attend Encore Coda again in the future? 


\section{APPENDIX C}

\section{INTERVIEWS}

\section{Camper Interview Questions}

Prompt: Tell me about your connection to camp encore coda and also any other camp experiences you may have had?

1) Why do you come to camp?

2) What makes camp camp?

3) How do you feel about having no cell phone, tv, or computer access at camp?

4) In what ways is camp different or similar to your learning experiences in school during the rest of the year?

5) How are your relationships with camp staff and faculty different or the same as with your music teachers in school?

6) What do you learn at camp?

7) What do you like about camp?

8) What do you dislike about camp?

9) To what extent does camp impact your musicianship?

10) Does camp impact you as a person in other ways besides musically?

11) Would you describe an outstanding experience you have had at camp?

12) Where do you see yourself in the future as far as future education or career?

\section{Staff/Faculty Interview Questions}

1) Tell me about you role at Camp Encore Coda?

2) How did you get connected with Encore Coda? (if not answered above)

4) Why did you choose to work at Encore Coda?

5) What is your musical background? 
6) How do you spend the rest of the year when you aren’t in camp?

7) What is the purpose of camp? Why do people attend camp? What makes it unique?

8) Why do YOU go to camp?

9) Are there any interesting or unique happenings at camp that an outsider would not see you could share?

10) What do you think about the structure of camp?

11) In regards to musical and social growth for the campers, are there any personal experiences or stories you could share that exemplify this, either as a camper yourself if you have attended or with campers you have observed or worked with?

12) Can you tell me about the overall community feel at camp?

13) What makes camp camp?

14) What impact does the isolation at camp from large population centers and technology have upon the attending campers?

15) Are there any other byproducts of this isolation? Do students have to adjust to these realities?

16) Describe your impact on the campers. Would you share any personal stories or experiences that would exemplify your assertion?

17) Could you describe one negative experience you have had at camp?

18) If you could change something about camp what would it be? 
APPENDIX D

OBSERVSATIONS TEMPLATE

\begin{tabular}{|l|l|}
\hline objective & subjective \\
\hline & \\
& \\
& \\
& \\
& \\
& \\
& \\
& \\
& \\
\end{tabular}

